Research Article

\title{
Improved Delay-Dependent Stability Criterion for Genetic Regulatory Networks with Interval Time-Varying Delays via New Lyapunov Functionals
}

\author{
Aphirak Boonpikum, ${ }^{1}$ Thongchai Botmart $\mathbb{D}^{1},{ }^{1}$ Piyapong Niamsup, ${ }^{2}$ and Wajaree Weera ${ }^{3}$ \\ ${ }^{1}$ Department of Mathematics, Khon Kaen University, Khon Kaen 40002, Thailand \\ ${ }^{2}$ Department of Mathematics, Chiang Mai University, Chiang Mai 50200, Thailand \\ ${ }^{3}$ Department of Mathematics, University of Phayao, Phayao 56000, Thailand
}

Correspondence should be addressed to Thongchai Botmart; thongbo@kku.ac.th

Received 23 June 2020; Accepted 22 September 2020; Published 21 October 2020

Academic Editor: Ramachandran Raja

Copyright (c) 2020 Aphirak Boonpikum et al. This is an open access article distributed under the Creative Commons Attribution License, which permits unrestricted use, distribution, and reproduction in any medium, provided the original work is properly cited.

\begin{abstract}
In this work, the stability analysis problem of the genetic regulatory networks (GRNs) with interval time-varying delays is presented. In the previous works, the constructions of Lyapunov functional have usually been in simple Lyapunov functional, augmented Lyapunov functional, and multiple integral Lyapunov functional. Therefore, we introduce new Lyapunov functionals expressed in terms of delay product functions. New delay-dependent sufficient conditions for the genetic regulatory networks (GRNs) are established in the terms of linear matrix inequalities (LMIS). In addition, a numerical example is provided to illustrate the effectiveness of the theoretical results.
\end{abstract}

\section{Introduction}

In recent years, genetic regulatory networks (GRNs) have achieved popularity in the biological and biomedical applications since it can effectively reflect the living organisms of molecular and cellular levels $[1,2]$. The researchers in various fields have wildly emphasized on GRNs. The study of living organism processes is one of the main challenges in our postgenomic era. Mathematical modelling of GRNs is a tool in the study of mechanism of control gene expression in an organism. The platforms of the studied model have various types such as Boolean models [3], differential equation model [4], stochastic equations $[5,6]$, and fractional-order dynamical systems [7]. In Boolean models, the expression of each gene is described to be either ON or OFF, no intermediate activity level is ever taken into consideration, and the state of gene is determined by the Boolean function of the states of other related genes [3]. In contrast, differential equation models described the continuous dynamical behaviors of GRNS between the concentration of gene product such as messenger ribonucleic acids (mRNAs) and proteins as the unknown functions. Consequently, differential equation models have drawn the attention of research studies to describe the gene regulatory process of organisms on the molecular level.

In experimental study in biochemistry, the gene expression was studied which consists of transcription and translation processes. As we known that the transcription and translation processes are slow reaction, time delay is inevitable in genetic regulatory network. In a biochemistry experiment on mice, it has been proved that there exists the time lag of about 15 minutes in the peaks between the mRNA molecules and the proteins of the gene Hes1 [8]. Thus, time delay has been attended in the study of mathematical modelling of GRNs.

Time delays in mathematical systems have received attention from researchers in the stability analysis which provide a poor performance or even instability of the relevant system [9-15]. In this reason, the stability analysis problems of genetic regulatory network with time delay have 
been reported in the literature $[6,8,16-28]$. They proposed new stability condition and reduced the possible conservativeness caused by the time delay. First, the local stability criteria for GRNs with constant delay were established in [8]. However, it is not sufficient to describe the behavior dynamic of nonlinear GRNs. The global asymptotic stability of GRN with SUM regulatory functions has been studied $[16,20,21,25,26]$. An increasing number of research studies about more complex modelled GRNs has been received attention in the following years, e.g., GRNs with distributed delayed [27], GRNs with nonlinear disturbance [17], leakage delay involved GRNS [22], and impulsive perturbation [24]. The stability problems of GRNs, such as stochastic stability [19], robust stability [18], exponential convergence analysis [28], and discrete-time stochastic stability $[6,23]$ have been also reported in the literature.

The stability analysis of systems with time-varying delays is the major problem of stability in systems. The conservatism stability condition and the maximum allowable delay bound have been recognized as the most important index. As we know that there are many methods to reduce the conservatism in stability criterions such as the development of the integral inequalities, i.e., the Jensens inequality, the Wirtinger-based inequality $[16,20]$, delay partitioning approach, and free-weighting matrix were used in [29], generalized zero equalities [30], and Lyapunov function approach [31]. As we know, in order to get the less conservative results, the main efforts were concentrated on two directions. One of the most popular approach is Lyapunov function. The construction of Lyapunov functions generally has two types, i.e., augmented Lyapunov functional approach (ALFA) and multiple integral Lyapunov functional approach (MILFA), which have been mainly utilized to propose new Lyapunov functionals [30-38]. The ALFA is more state information into the vector of the positive quadratic term, for example, $\xi^{T}(t) P \xi(t)$ with $\xi(t)$ including $x(t), y(t), f(x(t)), \int_{t-\tau_{1}}^{t} x(s) \mathrm{d} s$, and $\int_{t-\sigma_{1}}^{t} x(s) \mathrm{d} s$, while the MILFA is used in multiple integral terms of a positive quadratic term as a Lyapunov function. In $[32,33]$, they established a new Lyapunov functionals which are delay product-type functionals and lead to less conservative result in time-varying delay systems. Furthermore, the construction of MILFA also led to least conservatism and decision variables for uncertain systems with interval timevarying delay [30-38].

Motivated by the above discussion, the most important concerns are the improved stability conditions for genetic regulatory networks with time-varying delays. In this paper, we will present new Lyapunov functions which are extended from $[32,33]$, by adding two quadratic terms. One is double integral terms of a positive quadratic term and the other one is a quadratic term which does not need to meet positive definite and would relax the stability conditions. Moreover, the development of double integral inequality is utilized to derive sufficient conditions in terms of linear matrix inequalities (LMIS). Finally, a numerical example is provided to illustrate the effectiveness of the theoretical results.

Notations: throughout this paper, $\mathbb{R}^{n}$ and $\mathbb{R}^{m \times n}$ denote the $n$-dimensional Euclidean space and the set of all $m \times n$ real matrices, respectively. $\mathbb{S}_{+}^{n}$ denotes a set of positive definite matrices with $n \times n$ dimensions. $X>0$ means that the matrix $X$ is a real symmetric positive definite matrix. $I$ denotes the identity matrix with appropriate dimensions, $\operatorname{diag}\{. .$.$\} denotes block diagonal matrix. The superscript T$ denotes the matrix transposition. Col $\{.$.$\} denotes column$ matrix. The $*$ in the matrix represents the elements below the main diagonal of a symmetric matrix. $\operatorname{Sym}\{X\}$ indicates indicates $X+X^{T}$ For $X \in \mathbb{R}^{m \times n}$.

\section{Problem Formulations}

Genetic regulatory networks (GRNs) are composed of a number of genes that interact and regulate the expression of other genes by proteins (the gene product). The dynamic behavior of genetic regulatory networks with variable delays can be described by the following state equations [4]:

$$
\begin{aligned}
& \dot{m}_{i}(t)=-a_{i} m_{i}(t)+f_{i}\left(p_{1}(t-\sigma(t)), p_{2}(t-\sigma(t)),\right. \\
&\left.\ldots, p_{n}(t-\sigma(t))\right), \\
& \dot{p}_{i}(t)=-c_{i} p_{i}(t)+d_{i} m_{i}(t-\tau(t)),
\end{aligned}
$$

where $m_{i}(t)$ and $p_{i}(t)$ denote the concentration of mRNA and protein of the $i$ th node at time $t . a_{i}$ and $c_{i}$ are positive real numbers that present the degradation rates of mRNA and protein, respectively. $d_{i}$ is the translation rate. $\sigma(t)$ and $\tau(t)$ are transcriptional and translational delays, respectively, and function $f_{i}$ denotes the regulatory function or transcription function, which is generally nonlinear function but has a form of monotonicity of each variable. It is usual to assume that the regulatory function satisfies the following SUM logic [26]:

$$
\begin{aligned}
f_{i}( & \left.p_{1}(t-\sigma(t)), p_{2}(t-\sigma(t)), \ldots, p_{n}(t-\sigma(t))\right) \\
& =\sum_{j=1}^{n} b_{i j}\left(p_{j}(t-\sigma(t))\right) .
\end{aligned}
$$

And the function $b_{i j}\left(p_{j}(t)\right)$ is generally expressed by a monotonic function of the Hill form:

$$
b_{i j}\left(p_{j}(t)\right)=\left\{\begin{array}{cl}
\alpha_{i j} \frac{\left(p_{j}(t) / \beta_{j}\right)^{h_{j}}}{1+\left(p_{j}(t) / \beta_{j}\right)^{h_{j}}}, & \text { if transcription factor } j \text { is an activator of gene } i, \\
\alpha_{i j} 1 / 1+\left(p_{j}(t) / \beta_{j}\right)^{h_{j}}, & \text { if transcription factor } j \text { is a repressor of gene } i,
\end{array}\right.
$$


where $h_{j}$ is the Hill coefficient, $\beta_{j}$ is a positive scalar, and $\alpha_{i j}$ is a bounded constant denoting the dimensionless transcriptional rate of transcription factor $j$ to gene $i$. We note that

$$
\alpha_{i j} \frac{1}{1+\left(p_{j}(t) / \beta_{j}\right)^{h_{j}}}=\alpha_{i j}\left(1-\frac{\left(p_{j}(t) / \beta_{j}\right)^{h_{j}}}{1+\left(p_{j}(t) / \beta_{j}\right)^{h_{j}}}\right) .
$$

Thus, GRNs (1) can be rewritten as

$$
\begin{aligned}
& \dot{m}_{i}(t)=-a_{i} m_{i}(t)+\sum_{j=1}^{n} w_{i j} g_{j}\left(p_{j}(t-\sigma(t))\right)+J_{i}, \\
& \dot{p}_{i}(t)=-c_{i} p_{i}(t)+d_{i} m_{i}((t-\tau(t))),
\end{aligned}
$$

where $g_{j}(x)=\left(x / \beta_{i}\right)^{h_{j}} / 1+\left(x / \beta_{i}\right)^{h_{j}}$ is a monotonically increasing function, $J_{i}=\sum_{j \in V_{i}} \alpha_{i j}$, and $V_{i}$ is the set of all the transcription factor $j$ which is a repressor of gene $i$. The matrix $W=\left(w_{i j}\right) \in \mathbb{R}^{n \times n}$ is the coupling matrix of GRNs, which is defined as follows:

$$
w_{i j}\left(p_{j}(t)\right)= \begin{cases}\alpha_{i j}, & \text { if transcription factor } j \text { activates gene } i, \\ 0, & \text { if transcription factor } j \text { does not regulate gene } i, \\ -\alpha_{i j}, & \text { if transcription factor } j \text { represses gene } i .\end{cases}
$$

In compact matrix form, (5) can be written as

$$
\begin{aligned}
& \dot{m}(t)=-A m(t)+W g(p(t-\sigma(t)))+J, \\
& \dot{p}(t)=-C p(t)+D m(t-\tau(t)),
\end{aligned}
$$

where

$$
\begin{aligned}
m(t) & =\left[m_{1}(t), m_{2}(t), \ldots, m_{n}(t)\right]^{T}, \\
p(t) & =\left[p_{1}(t), p_{2}(t), \ldots, p_{n}(t)\right]^{T}, \\
g(p(t)) & =\left[g_{1}\left(p_{1}(t)\right), g_{2}\left(p_{2}(t)\right), \ldots, g_{n}\left(p_{n}(t)\right)\right]^{T}, \\
J & =\left[j_{1}, j_{2}, \ldots, j_{n}\right]^{T}, \\
A & =\operatorname{diag}\left\{k_{m 1}, k_{m 2}, \ldots, k_{m n}\right\}, \\
C & =\operatorname{diag}\left\{k_{p 1}, k_{p 2}, \ldots, k_{p n}\right\}, \\
D & =\operatorname{diag}\left\{r_{1}, r_{2}, \ldots, r_{n}\right\} .
\end{aligned}
$$

Let $\left(m^{*}, p^{*}\right)$ be the equilibrium point of GRNs (7), where $m^{*}=\left[m_{1}^{*}, m_{2}^{*}, \ldots, m_{n}^{*}\right]^{T}$ and $p^{*}=\left[p_{1}^{*}, p_{2}^{*}\right.$, $\left.\ldots, p_{n}^{*}\right]^{T}$; then, we obtain

$$
\begin{aligned}
-A m^{*}+W g\left(p^{*}\right) & =0, \\
-C p^{*}+D m^{*} & =0 .
\end{aligned}
$$

Shifting equilibrium point $\left(m^{*}, p^{*}\right)$ to the origin, using the following transformation $x(t)=m(t)-m^{*}$ and $y(t)=p(t)-p^{*}$, model (7) can be transformed into the following form:

$$
\begin{aligned}
& \dot{x}(t)=-A x(t)+W f(y(t-\sigma(t))), \\
& \dot{y}(t)=-C y(t)+D x(t-\tau(t)),
\end{aligned}
$$

where $f(y(t))=g\left(p(t)+p^{*}\right)-g\left(p^{*}\right)$. Since the function $g$ is monotonically increasing function with saturation, it satisfies that, for all $a, b \in \mathbb{R}$ with $a \neq b$,

$$
0 \leq \frac{g(a)-g(b)}{a-b} \leq k .
$$

When $g(\cdot)$ is differentiable, the above inequality is equivalent to $0 \leq g d(a) / d(a) \leq k$. From the relationship of $f(\cdot)$ and $g(\cdot)$, we know that $f(\cdot)$ satisfies the sector condition [39], or equivalently

$$
f(a)(f(a)-k a) \leq 0 .
$$

Assumption 1. The assumptions of time-delay conditions are

$$
\begin{aligned}
& 0<\tau_{1} \leq \tau(t) \leq \tau_{2}, \\
& 0<\sigma_{1} \leq \sigma(t) \leq \sigma_{2}, \\
& 0<\dot{\tau}(t) \leq \tau_{d} \text { and } 0<\dot{\sigma}(t) \leq \sigma_{d},
\end{aligned}
$$

where $0<\tau_{1} \leq \tau_{2}, 0<\sigma_{1} \leq \sigma_{2}, \tau_{d}>0$, and $\sigma_{d}>0$.

Assumption 2. Let $g_{i}: \mathbb{R} \longrightarrow \mathbb{R}, i=1, \ldots, n$, be monotonically increasing functions with saturation and moreover satisfy

$$
\begin{aligned}
0 & \leq \frac{g_{i}(x)}{x} \leq l_{i}, l_{i}=\max _{u \geq 0} f_{j}^{\prime}(u) \\
& =\frac{\left(h_{i}-1\right)^{h_{i}-1 / h_{i}}\left(h_{i}+1\right)^{h_{i}-1 / h_{i}}}{4 b_{i} h_{i}} .
\end{aligned}
$$

Remark 1. The modelling of GRNs is largely dependent on powerful tools of mathematics theory. The differential equation model has drawn a lot of research attention since variables in gene dynamics are usually the concentrations of gene product (messenger ribonucleic acids (mRNAs) and proteins). It has been shown that the time delays may play an important role in the predictions of the dynamics of the mRNA and protein concentrations; GRNs models without consideration of time delays may provide wrong predictions. Therefore, it is significant to study the stability of delayed GRNs and sufficient stability conditions. However, the conservatism stability condition and the maximum allowable delay bound have been recognized as the most important index. Therefore, in this research, we have improved delay dependent stability criterion for genetic regulatory 
network (GRNs) with interval-time varying delays via new Lyapunov function by constructing a new Lyapunov functionals in Section 4. In addition, we have established one integral and double integral inequalities to estimate. Therefore, the results had been established that the stability criterion lead to less conservativeness.
To proceed, the following lemmas are introduced which will be useful for further derivations.

Lemma 1 (Jensen integral inequalities, see [40]). Let $w:[a, b] \longrightarrow \mathbb{R}^{n}$ be a differentiable function. For any given matrix $R>0$ and $a \leq s \leq b$, the following inequalities hold:

$$
\begin{gathered}
(b-a) \int_{a}^{b} \dot{w}^{T}(s) R \dot{w}(s) d s \geq\left(\int_{a}^{b} \dot{w}(s) \mathrm{d} s\right)^{T} R\left(\int_{a}^{b} \dot{w}(s) \mathrm{d} s\right), \\
\frac{(b-a)^{2}}{2} \int_{a}^{b} \int_{s}^{b} \dot{w}^{T}(u) R \dot{w}(u) \mathrm{d} u \mathrm{~d} s \geq\left(\int_{a}^{b} \int_{s}^{b} \dot{w}(u) \mathrm{d} u \mathrm{~d} s\right)^{T} R\left(\int_{a}^{b} \int_{s}^{b} \dot{w}(u) \mathrm{d} u \mathrm{~d} s\right) .
\end{gathered}
$$

Lemma 2 (Wirtinger-based integral inequality, see [41]). Let $w:[a, b] \longrightarrow \mathbb{R}^{n}$ be a differentiable function. For any given matrix $R>0$ and $a \leq s \leq b$, the following inequality holds:

$$
(b-a) \int_{a}^{b} w^{T}(s) R \dot{w}(s) \mathrm{d} s \geq \chi_{1}^{T} R \chi_{1}+3 \chi_{2}^{T} R \chi_{2}+5 \chi_{3}^{T} R \chi_{3},
$$

where

$$
\begin{aligned}
\chi_{1}= & w(b)-w(a), \\
\chi_{2}= & w(b)+w(a)-\frac{2}{b-a} \int_{a}^{b} w(s) \mathrm{d} s, \\
\chi_{3}= & w(b)-w(a)+\frac{6}{b-a} \int_{a}^{b} w(s) \mathrm{d} s \\
& -\frac{12}{(b-a)^{2}} \int_{a}^{b} \int_{s}^{b} w(u) \mathrm{d} u \mathrm{~d} s .
\end{aligned}
$$

Lemma 3 (relaxed double integral inequality, see [35]). Let $w:[a, b] \longrightarrow \mathbb{R}^{n}$ be a differentiable function. For any given matrix $R>0$ and $a \leq s \leq b$, the following inequality holds:

$$
\int_{a}^{b} \int_{s}^{b} \dot{w}^{T}(u) R \dot{w}(u) \mathrm{d} u \mathrm{~d} s \geq 2 \chi_{1}^{T} R \chi_{1}+4 \chi_{2}^{T} R \chi_{2},
$$

where

$$
\begin{aligned}
& \chi_{1}=w(b)-\frac{1}{b-a} \int_{a}^{b} w(s) \mathrm{d} s \\
& \chi_{2}=w(b)+\frac{2}{b-a} \int_{a}^{b} w(s) \mathrm{d} s-\frac{6}{(b-a)^{2}} \int_{a}^{b} \int_{s}^{b} w(u) \mathrm{d} u \mathrm{~d} s .
\end{aligned}
$$

Lemma 4 (Schur complement, see [42]). For a symmetric matrix $S=\left[\begin{array}{cc}S_{11} & S_{12} \\ * & S_{22}\end{array}\right]$, the following conditions are
equivalent:
(a) $S<0$
(b) $S_{11}<0$ and $S_{22}-S_{12}^{T} S_{11}^{-1} S_{12}<0$
(c) $S_{22}<0$ and $S_{11}-S_{12} S_{22}^{-1} S_{12}^{T}<0$

\section{Improved Integral Inequalities}

In this section, we propose the following results which will be used to the development of the inequality.

Lemma 5 (extended relaxed one integral inequality, see [42]). Let $w:\left[\alpha_{1}, \alpha_{2}\right] \longrightarrow \mathbb{R}^{n}$ be a differentiable function, for a time-varying scalar $\alpha(t) \in\left[\alpha_{1}, \alpha_{2}\right]$, symmetric matrices $\widetilde{R}_{i}=\operatorname{diag}\left\{R_{i}, 3 R_{i}\right\}$, with $R_{i}>0$, and any matrices $S_{i} \in \mathbb{R}^{2 n \times 2 n}$, $i=1,2$, the following integral inequality holds:

$$
\begin{aligned}
& \int_{t-\alpha(t)}^{t-\alpha_{1}} \dot{w}^{T}(s) R_{1} \dot{w}(s) \mathrm{d} s+\int_{t-\alpha(t)}^{t-\alpha_{1}(t)} \dot{w}^{T}(s) R_{2} \dot{w}(s) \mathrm{d} s \\
& \geq \frac{1}{\alpha_{21}}\left[\begin{array}{c}
E_{1} \\
E_{2}
\end{array}\right]^{T}\left(\left[\begin{array}{cc}
(1+\beta) \widetilde{R}_{1} & \beta S_{1}+\alpha S_{2} \\
(1+\alpha) \widetilde{R}_{2}
\end{array}\right]-\left[\begin{array}{cc}
\beta S_{2} \widetilde{R}_{2}^{-1} S_{2}^{T} & 0 \\
0 & \alpha S_{1}^{T} \widetilde{R}_{1}^{-1} S_{1}
\end{array}\right]\right)\left[\begin{array}{l}
E_{1} \\
E_{2}
\end{array}\right],
\end{aligned}
$$


where

$$
\begin{aligned}
\alpha_{21} & =\alpha_{2}-\alpha_{1}, \\
\alpha & =\frac{\alpha(t)-\alpha_{1}}{\alpha_{21}}=\frac{\alpha_{1}(t)}{\alpha_{21}}, \\
\beta & =\frac{\alpha_{2}-\alpha(t)}{\alpha_{21}}=\frac{\alpha_{2}(t)}{\alpha_{21}}, \\
E_{1} & =\left[\begin{array}{l}
w\left(t-\alpha_{1}\right)-w(t-\alpha(t)) \\
w\left(t-\alpha_{1}\right)+w(t-\alpha(t))-\frac{2}{\alpha_{1}(t)} \int_{t-\alpha(t)}^{t-\alpha_{1}} w(s) \mathrm{d} s
\end{array}\right], \\
E_{2} & =\left[\begin{array}{c}
w(t-\alpha(t))-w\left(t-\alpha_{2}\right) \\
w(t-\alpha(t))+w\left(t-\alpha_{2}\right)-\frac{2}{\alpha_{2}(t)} \int_{t-\alpha_{2}}^{t-\alpha(t)} w(s) \mathrm{d} s
\end{array}\right] .
\end{aligned}
$$

Proof. By setting a function $f(s, a, b)=2 s-b-a / b-a$, where $a$ and $b$ are constants, then the following equations are derived:

$$
\begin{aligned}
\int_{a}^{b} \dot{w}(s) \mathrm{d} s & =w(b)-w(a) \\
\int_{a}^{b} f(s, a, b) \dot{w}(s) \mathrm{d} s & =w(b)+w(a)-\frac{2}{b-a} \int_{b}^{a} w(s) \mathrm{d} s \\
\int_{a}^{b} f(s, a, b) \mathrm{d} s & =0 \\
\int_{a}^{b} f^{2}(s, a, b) \mathrm{d} s & =\frac{b-a}{3} .
\end{aligned}
$$

Furthermore, based on Schur complement, for symmetric matrices $R_{i}>0, i=1,2$, and any matrices $X_{j}$, $j=1,2,3,4$, with appropriate dimensions, the following inequalities hold:

$$
\begin{aligned}
& {\left[\begin{array}{ccc}
X_{1} R_{1}^{-1} X_{1}^{-1} & X_{1} R_{1}^{-1} X_{2}^{-1} & X_{1} \\
& X_{2} R_{1}^{-1} X_{2}^{-1} & X_{2} \\
* & R_{1}
\end{array}\right] \geq 0,} \\
& {\left[\begin{array}{ccc}
X_{3} R_{2}^{-1} X_{3}^{-1} & X_{3} R_{2}^{-1} X_{4}^{-1} & X_{3} \\
& X_{4} R_{2}^{-1} X_{4}^{-1} & X_{4} \\
& * & R_{2}
\end{array}\right] \geq 0,}
\end{aligned}
$$

which lead to

$$
\begin{aligned}
\Pi_{1}= & \int_{t-\alpha(t)}^{t-\alpha_{1}}\left[\begin{array}{c}
g \\
\lambda_{1} g \\
\dot{w}(s)
\end{array}\right]^{T}\left[\begin{array}{ccc}
X_{1} R_{1}^{-1} X_{1}^{-1} & X_{1} R_{1}^{-1} X_{2}^{-1} & X_{1} \\
& X_{2} R_{1}^{-1} X_{2}^{-1} & X_{2} \\
* & R_{1}
\end{array}\right] \\
& \cdot\left[\begin{array}{c}
g \\
\lambda_{1} g \\
\dot{w}(s)
\end{array}\right] \mathrm{d} s \geq 0, \\
\Pi_{2}= & {\left[\begin{array}{ccc}
t-\alpha(t) \\
t-\alpha_{2} \\
\lambda_{2} g \\
\dot{w}(s)
\end{array}\right]^{T}\left[\begin{array}{ccc}
X_{3} R_{2}^{-1} X_{3}^{-1} & X_{3} R_{2}^{-1} X_{4}^{-1} & X_{3} \\
X_{4} R_{2}^{-1} X_{4}^{-1} & X_{4} \\
* & R_{2}
\end{array}\right] } \\
& \cdot\left[\begin{array}{c}
g \\
\lambda_{2} g \\
\dot{w}(s)
\end{array}\right]
\end{aligned}
$$

where $g=\left[E_{1}^{T}, E_{2}^{T}\right]^{T}, \quad \lambda_{1}=f\left(s, t-\alpha_{1}, t-\alpha(t)\right), \quad$ and $\lambda_{2}=f\left(s, t-\alpha(t), t-\alpha_{2}\right)$.

Define matrices $X_{i}, i=1,2,3,4$, as follows:

$$
\begin{aligned}
& X_{1}=-\frac{1}{\alpha_{21}}\left[\begin{array}{c}
R_{1} \\
0 \\
L_{1}
\end{array}\right], \\
& X_{2}=-\frac{1}{\alpha_{21}}\left[\begin{array}{c}
0 \\
3 R_{1} \\
L_{2}
\end{array}\right], \\
& X_{3}=-\frac{1}{\alpha_{21}}\left[\begin{array}{c}
L_{3} \\
R_{2} \\
0
\end{array}\right], \\
& X_{4}=-\frac{1}{\alpha_{21}}\left[\begin{array}{c}
L_{4} \\
0 \\
3 R_{2}
\end{array}\right],
\end{aligned}
$$

where $L_{i}, i=1,2,3,4$, are appropriate dimensional matrices, $S_{1}=\left[L_{1}, L_{2}\right]^{T}$ and $S_{2}=\left[L_{3}, L_{4}\right]$. Then, the following equivalent relations are taken into account similar to [42]: 


$$
\begin{aligned}
& \int_{t-\alpha(t)}^{t-\alpha_{1}}\left[\begin{array}{c}
g \\
\lambda_{1} g
\end{array}\right]^{T}\left[\begin{array}{ll}
X_{1} R_{1}^{-1} X_{1}^{-1} & X_{2} R_{1}^{-1} X_{2}^{-1} \\
X_{2} R_{1}^{-1} X_{2}^{-1}
\end{array}\right]\left[\begin{array}{c}
g \\
\lambda_{1} g
\end{array}\right] \mathrm{d} s=\frac{\alpha_{1}(t)}{\alpha_{21}^{2}}\left[\begin{array}{c}
E_{1} \\
E_{2}
\end{array}\right]^{T}\left[\begin{array}{ccc}
R_{1} & 0 & L_{1}^{T} \\
0 & 3 R_{1} & L_{2}^{T} \\
L_{1} & L_{2} & L_{1} R_{1}^{-1} L_{1}^{T}+L_{2}\left(3 R_{1}\right)^{-1} L_{2}^{T}
\end{array}\right]\left[\begin{array}{c}
E_{1} \\
E_{2}
\end{array}\right] \\
& =\frac{1}{\alpha_{21}}\left[\begin{array}{c}
E_{1} \\
E_{2}
\end{array}\right]^{T}\left(\frac{\alpha_{1}(t)}{\alpha_{21}}\right)\left[\begin{array}{cc}
\widetilde{R}_{1} & S_{1} \\
* & S_{1}^{T} \widetilde{R}_{1}^{-1} S_{1}
\end{array}\right]\left[\begin{array}{c}
E_{1} \\
E_{2}
\end{array}\right], \\
& 2 \int_{t-\alpha(t)}^{t-\alpha_{1}}\left[\begin{array}{c}
g \\
\lambda_{1} g
\end{array}\right]^{T}\left[\begin{array}{c}
X_{1} \\
X_{2}
\end{array}\right] \dot{x}(s) \mathrm{d} s=-\frac{1}{\alpha_{21}}\left[\begin{array}{c}
E_{1} \\
E_{2}
\end{array}\right]^{T}\left[\begin{array}{ccc}
2 R_{1} & 0 & L_{1}^{T} \\
0 & 6 R_{1} & L_{2}^{T} \\
L_{1} & L_{2} & 0
\end{array}\right]\left[\begin{array}{c}
E_{1} \\
E_{2}
\end{array}\right] \\
& =-\frac{1}{\alpha_{21}}\left[\begin{array}{c}
E_{1} \\
E_{2}
\end{array}\right]^{T}\left[\begin{array}{cc}
2 \widetilde{R}_{1} & S_{1} \\
* & 0
\end{array}\right]\left[\begin{array}{c}
E_{1} \\
E_{2}
\end{array}\right] \text {, } \\
& \int_{t-\alpha(t)}^{t-\alpha_{1}}\left[\begin{array}{c}
g \\
\lambda_{1} g
\end{array}\right]^{T}\left[\begin{array}{cc}
X_{3} R_{2}^{-1} X_{3}^{-1} & X_{3} R_{2}^{-1} X_{4}^{-1} \\
& X_{4} R_{2}^{-1} X_{4}^{-1}
\end{array}\right]\left[\begin{array}{c}
g \\
\lambda_{2} g
\end{array}\right] \mathrm{d} s=\frac{\alpha_{2}(t)}{\alpha_{21}}\left[\begin{array}{c}
E_{1} \\
E_{2}
\end{array}\right]^{T}\left[\begin{array}{ccc}
L_{3} R_{2}^{-1} L_{3}^{T}+L_{4}\left(3 \widetilde{R}_{2}\right)^{-1} L_{4}^{T} & L_{3} & L_{4} \\
L_{3}^{T} & R_{2} & 0 \\
L_{4}^{T} & 0 & 3 R_{2}
\end{array}\right]\left[\begin{array}{c}
E_{1} \\
E_{2}
\end{array}\right] \\
& =\frac{1}{\alpha_{21}}\left[\begin{array}{c}
E_{1} \\
E_{2}
\end{array}\right]^{T}\left(\frac{\alpha_{2}(t)}{\alpha_{21}}\right)\left[\begin{array}{cc}
S_{2} \widetilde{R}_{2}^{-1} S_{2}^{T} & S_{2} \\
* & \widetilde{R}_{2}
\end{array}\right]\left[\begin{array}{c}
E_{1} \\
E_{2}
\end{array}\right], \\
& 2 \int_{t-\alpha_{2}}^{t-\alpha(t)}\left[\begin{array}{c}
g \\
\lambda_{2} g
\end{array}\right]^{T}\left[\begin{array}{c}
X_{3} \\
X_{4}
\end{array}\right] \dot{x}(s) \mathrm{d} s=-\frac{1}{\alpha_{21}}\left[\begin{array}{c}
E_{1} \\
E_{2}
\end{array}\right]^{T}\left[\begin{array}{ccc}
0 & L_{3} & L_{4} \\
L_{3}^{T} & 2 R_{2} & 0 \\
L_{4}^{T} & 0 & 6 R_{2}
\end{array}\right]\left[\begin{array}{c}
E_{1} \\
E_{2}
\end{array}\right] \\
& =-\frac{1}{\alpha_{21}}\left[\begin{array}{c}
E_{1} \\
E_{2}
\end{array}\right]^{T}\left[\begin{array}{cc}
2 \widetilde{R}_{2} & S_{2} \\
* & 0
\end{array}\right]\left[\begin{array}{c}
E_{1} \\
E_{2}
\end{array}\right] .
\end{aligned}
$$

To sum up with multiplication, we have

$$
\begin{aligned}
0 \leq \Pi_{1}+\Pi_{2}= & \int_{t-\alpha(t)}^{t-\alpha_{1}} \dot{w}(s) R_{1} \dot{w}(s) \mathrm{d} s+\int_{t-\alpha_{2}}^{t-\alpha(t)} \dot{w}(s) R_{2} \dot{w}(s) \mathrm{d} s\left[\begin{array}{c}
E_{1} \\
E_{2}
\end{array}\right] \\
& -\frac{1}{\alpha_{21}}\left[\begin{array}{c}
E_{1} \\
E_{2}
\end{array}\right]^{T}\left\{\left[\begin{array}{cc}
(1+(1-\alpha)) \widetilde{R}_{1} & (1-\alpha) S_{1}+\alpha S_{2} \\
* & (1+\alpha) \widetilde{R}_{2}
\end{array}\right]-\left[\begin{array}{cc}
(1-\alpha) S_{2} \widetilde{R}_{2}^{-1} S_{2}^{T} & 0 \\
0 & \alpha S_{1}^{T} \widetilde{R}_{1}^{-1} S_{1}
\end{array}\right]\right\}\left[\begin{array}{c}
E_{1} \\
E_{2}
\end{array}\right] .
\end{aligned}
$$


Thus,

$$
\Pi_{1}+\Pi_{2} \geq \frac{1}{\alpha_{21}}\left[\begin{array}{c}
E_{1} \\
E_{2}
\end{array}\right]^{T}\left\{\left[\begin{array}{cc}
(1+(1-\alpha)) \widetilde{R}_{1} & (1-\alpha) S_{1}+\alpha S_{2} \\
* & (1+\alpha) \widetilde{R}_{2}
\end{array}\right]-\left[\begin{array}{cc}
(1-\alpha) S_{2} \widetilde{R}_{2}^{-1} S_{2}^{T} & 0 \\
0 & \alpha S_{1}^{T} \widetilde{R}_{1}^{-1} S_{1}
\end{array}\right]\right\}\left[\begin{array}{l}
E_{1} \\
E_{2}
\end{array}\right]
$$

This completes the proof inequality (20).

Remark 2. Consider the inequality in Lemma 4 [42] can be written in the following form:

$$
\begin{aligned}
& \int_{t-h(t)}^{t} \dot{w}^{T}(s) R \dot{w}(s) \mathrm{d} s+\int_{t-h}^{t-h(t)} \dot{w}^{T}(s) R \dot{w}(s) \mathrm{d} s \\
& \geq \frac{1}{h}\left[\begin{array}{c}
E_{1} \\
E_{2}
\end{array}\right]^{T}\left[\begin{array}{cc}
\widetilde{R}+\beta\left(\widetilde{R}-S \widetilde{R}^{-1} S^{T}\right) & S \\
* & \widetilde{R}+\alpha\left(\widetilde{R}-S^{T} \widetilde{R}^{-1} S\right)
\end{array}\right] \\
& \cdot\left[\begin{array}{c}
E_{1} \\
E_{2}
\end{array}\right] .
\end{aligned}
$$

The advantage of Lemma 5 can be concluded as follows:

(1) Equation (20) can be reduced to Lemma 4 [42] when $S_{1}=S_{2}$ and the lower bound of time delay is zeros, i.e., $\alpha(t) \in[0, h]$. However, Lemma 4 [42] cannot be used to estimate in the time-delay interval $\alpha(t) \in\left[\alpha_{1}, \alpha_{2}\right]$

(2) The matrix $S$ in [42] can be written as $\beta S_{1}+\alpha S_{2}$ which leads to exactitude in the estimation of equation (20).

(3) The matrices $R_{1}=R_{2}$ can deal with Lemma 4 [42]. Lemma 5 can be applied in case of the different matrices, in which Lemma 4 [42] cannot be estimated in this case.

Remark 3. From the estimation of Corollary 5 [41] and reciprocally convex combination lemma [43], the estimation of integral (20) is as follows:

$$
\begin{aligned}
& \int_{t-\alpha(t)}^{t-\alpha_{1}} \dot{w}^{T}(s) R_{1} \dot{w}(s) \mathrm{d} s+\int_{t-\alpha_{2}}^{t-\alpha(t)} \dot{w}^{T}(s) R_{2} \dot{w}(s) \mathrm{d} s \\
& \geq \frac{1}{\alpha_{21}}\left[\begin{array}{c}
E_{1} \\
E_{2}
\end{array}\right]^{T}\left[\begin{array}{cc}
\widetilde{R}_{1} & S \\
* & \widetilde{R}_{2}
\end{array}\right]\left[\begin{array}{l}
E_{1} \\
E_{2}
\end{array}\right] .
\end{aligned}
$$

When $\left[\begin{array}{cc}\widetilde{R}_{1} & S \\ * & \widetilde{R}_{2}\end{array}\right]>0$, compared with inequality (20), two aspects can be shown:

(1) The requirement $\left[\begin{array}{cc}\widetilde{R}_{1} & S \\ * & \widetilde{R}_{2}\end{array}\right]>0$ and the matrix $S$ which is relaxed to $\beta S_{1}+\alpha S_{2}$ is substituted in (20).

(2) The estimation gap between (20) and (30), which is calculated from the right-hand side of (20) and (30), is, respectively, defined by $\Gamma_{1}$ and $\Gamma_{2}$; then,

$$
\Gamma_{1}-\Gamma_{2}=\left[\begin{array}{cc}
\beta\left(\widetilde{R}_{1}-S_{2} \widetilde{R}_{2}^{-1} S_{2}^{T}\right) & 0 \\
0 & \alpha\left(\widetilde{R}_{2}-S_{1} \widetilde{R}_{1}^{-1} S_{1}^{T}\right)
\end{array}\right] .
$$

(i) By using Schur complement, then $\Gamma_{1}-\Gamma_{2}>0$. This can be shown that an upper bound of inequality (20) is more than (30). So, we can see that (20) is less conservative than (30).

Lemma 6 (extended relaxed double integral inequality). Let $w:\left[\alpha_{1}, \alpha_{2}\right] \longrightarrow \mathbb{R}^{n}$ be a differentiable function, for a timevarying scalar $\alpha(t) \in\left[\alpha_{1}, \alpha_{2}\right]$, symmetric matrices $\widetilde{R}_{i}=\operatorname{diag}\left\{2 R_{i}, 4 R_{i}\right\}$, with $R_{i}>0, i=1,2$ and any matrices $S$, and the following integral inequality holds:

$$
\begin{aligned}
& \int_{t-\alpha(t)}^{t-\alpha_{1}} \int_{\theta}^{t-\alpha_{1}} w^{T}(s) R_{1} \dot{w}(s) \mathrm{d} s \mathrm{~d} \theta \\
& \quad+\int_{t-\alpha_{2}}^{t-\alpha(t)} \int_{\theta}^{t-\alpha(t)} w^{T}(s) R_{2} \dot{w}(s) \mathrm{d} s \mathrm{~d} \theta \\
& \geq\left[\begin{array}{c}
F_{1} \\
F_{2}
\end{array}\right]^{T}\left(\left[\begin{array}{cc}
\widetilde{R}_{1} & 0 \\
0 & \widetilde{R}_{2}
\end{array}\right]-\left[\begin{array}{cc}
\alpha_{2}^{2}(t) S_{2} \widetilde{R}_{2}^{-1} S_{2}^{T} & 0 \\
0 & \alpha_{1}^{2}(t) S_{1}^{T} \widetilde{R}_{1}^{-1} S_{1}
\end{array}\right]\right) \\
& \quad \cdot\left[\begin{array}{c}
F_{1} \\
F_{2}
\end{array}\right],
\end{aligned}
$$

where 


$$
\begin{aligned}
\alpha_{21} & =\alpha_{2}-\alpha_{1}, \\
\alpha_{1}(t) & =\alpha(t)-\alpha_{1}, \\
\alpha_{2}(t) & =\alpha_{2}-\alpha(t), \\
F_{1} & =\left[\begin{array}{l}
w\left(t-\alpha_{1}\right)-\frac{1}{\alpha_{1}(t)} \int_{t-\alpha(t)}^{t-\alpha_{1}} w(s) \mathrm{d} s \\
\left.w\left(t-\alpha_{1}\right)+\frac{2}{\alpha_{1}(t)} \int_{t-\alpha(t)}^{t-\alpha_{1}} w(s) \mathrm{d} s-\frac{6}{\alpha_{1}^{2}(t)} \int_{t-\alpha(t)}^{t-\alpha_{1}} \int_{\theta}^{t-\alpha_{1}} w(s) \mathrm{d} s\right]
\end{array}\right], \\
F_{2}= & {\left[\begin{array}{l}
w(t-\alpha(t))-\frac{1}{\alpha_{2}(t)} \int_{t-\alpha_{2}}^{t-\alpha(t)} w(s) \mathrm{d} s \\
\left.w(t-\alpha(t))+\frac{2}{\alpha_{2}(t)} \int_{t-\alpha_{2}}^{t-\alpha(t)} w(s) \mathrm{d} s-\frac{6}{\alpha_{2}^{2}(t)} \int_{t-\alpha_{2}}^{t-\alpha(t)} \int_{\theta}^{t-\alpha(t)} w(s) \mathrm{d} s \mathrm{~d} \theta\right]
\end{array}\right.}
\end{aligned}
$$

Proof. By setting a function $f(s, a, b)=3 s-a-2 b / 2(b-a)$, where $a$ and $b$ are constants, then the following equations are derived:

$$
\begin{aligned}
\int_{a}^{b} \int_{\theta}^{b} \dot{w}(s) \mathrm{d} s \mathrm{~d} \theta & =(b-a)\left(w(b)-\frac{1}{b-a} \int_{a}^{b} w(s) \mathrm{d} s\right) \\
\int_{a}^{b} \int_{\theta}^{b} f(s, a, b) \dot{w}(s) \mathrm{d} s \mathrm{~d} \theta & =\frac{b-a}{2}\left(w(b)+\frac{2}{b-a} \int_{a}^{b} w(s) \mathrm{d} s-\frac{6}{(b-a)^{2}} \int_{a}^{b} \int_{\theta}^{b} w(s) \mathrm{d} s \mathrm{~d} \theta\right), \\
\int_{a}^{b} \int_{\theta}^{b} f(s, a, b) \mathrm{d} s \mathrm{~d} \theta & =0, \\
\int_{a}^{b} \int_{\theta}^{b} f^{2}(s, a, b) \mathrm{d} s \mathrm{~d} \theta & =\frac{(b-a)^{2}}{16} .
\end{aligned}
$$

Furthermore, based on Schur complement, for symmetric matrices $R_{i}>0, i=1,2$, and any matrices $X_{j}$, $j=1,2,3,4$, with appropriate dimensions, the following inequalities hold:

$$
\begin{gathered}
{\left[\begin{array}{ccc}
X_{1} R_{1}^{-1} X_{1}^{-1} & X_{1} R_{1}^{-1} X_{2}^{-1} & X_{1} \\
& X_{2} R_{1}^{-1} X_{2}^{-1} & X_{2} \\
* & R_{1}
\end{array}\right] \geq 0,} \\
{\left[\begin{array}{ccc}
X_{3} R_{2}^{-1} X_{3}^{-1} & X_{3} R_{2}^{-1} X_{4}^{-1} & X_{3} \\
& X_{4} R_{2}^{-1} X_{4}^{-1} & X_{4} \\
* & R_{2}
\end{array}\right] \geq 0 .}
\end{gathered}
$$

$$
\begin{aligned}
& \Pi_{1}=\int_{t-\alpha(t)}^{t-\alpha_{1}} * \int_{\theta}^{t-\alpha_{1}}\left[\begin{array}{c}
g \\
\lambda_{1} g \\
\dot{w}(s)
\end{array}\right]^{T} \\
& {\left[\begin{array}{ccc}
X_{1} R_{1}^{-1} X_{1}^{-1} & X_{1} R_{1}^{-1} X_{2}^{-1} & X_{1} \\
* & X_{2} R_{1}^{-1} X_{2}^{-1} & X_{2} \\
* & * & R_{1}
\end{array}\right]\left[\begin{array}{c}
g \\
\lambda_{1} g \\
\dot{w}(s)
\end{array}\right] \mathrm{d} s \mathrm{~d} \theta \geq 0,} \\
& \Pi_{2}=\int_{t-\alpha_{2}}^{t-\alpha(t)} \int_{\theta}^{t-\alpha(t)}\left[\begin{array}{c}
g \\
\lambda_{2} g \\
\dot{w}(s)
\end{array}\right]^{T} \\
& {\left[\begin{array}{ccc}
X_{3} R_{2}^{-1} X_{3}^{-1} & X_{3} R_{2}^{-1} X_{4}^{-1} & X_{3} \\
* & X_{4} R_{2}^{-1} X_{4}^{-1} & X_{4} \\
* & * & R_{2}
\end{array}\right]\left[\begin{array}{c}
g \\
\lambda_{2} g \\
\dot{w}(s)
\end{array}\right] \mathrm{d} s \mathrm{~d} \theta \geq 0,}
\end{aligned}
$$


where $\quad g=\left[F_{1}^{T}, F_{2}^{T}\right]^{T}, \quad \lambda_{1}=f\left(s, t-\alpha(t), t-\alpha_{1}\right), \quad$ and $\lambda_{2}=f\left(s, t-\alpha_{2}, t-\alpha(t)\right)$. Define matrices $X_{i}, i=1,2,3,4$, as follows:

$$
\begin{aligned}
& X_{1}=-\operatorname{col}\left\{\frac{2}{\alpha_{1}(t)} R_{1}, 0, L_{1}\right\}, \\
& X_{2}=-\operatorname{col}\left\{0, \frac{8}{\alpha_{1}(t)} R_{1}, 2 L_{2}\right\}, \\
& X_{3}=-\operatorname{col}\left\{L_{3}, \frac{2}{\alpha_{2}(t)} R_{2}, 0\right\}, \\
& X_{4}=-\operatorname{col}\left\{2 L_{4}, 0, \frac{8}{\alpha_{2}(t)} R_{2}\right\},
\end{aligned}
$$

where $L_{i}, i=1,2,3,4$, are appropriate dimensional matrices, $S_{1}=\left[L_{1}, L_{2}\right]^{T}$ and $S_{2}=\left[L_{3}, L_{4}\right]$. Carrying out simple algebraic calculation, then

$$
\begin{aligned}
& \int_{t-\alpha(t)}^{t-\alpha_{1}} \int_{\theta}^{t-\alpha_{1}}\left[\begin{array}{c}
g \\
\lambda_{1} g
\end{array}\right]^{T}\left[\begin{array}{ll}
X_{1} R_{1}^{-1} X_{1}^{-1} & X_{2} R_{1}^{-1} X_{2}^{-1} \\
& X_{2} R_{1}^{-1} X_{2}^{-1}
\end{array}\right]\left[\begin{array}{c}
g \\
\lambda_{1} g
\end{array}\right] \mathrm{d} s \mathrm{~d} \theta \\
& =\left[\begin{array}{l}
F_{1} \\
F_{2}
\end{array}\right]^{T}\left[\begin{array}{ccc}
2 R_{1} & 0 & \alpha_{1}(t) L_{1}^{T} \\
0 & 4 R_{1} & \alpha_{1}(t) L_{2}^{T} \\
\alpha_{1}(t) L_{1} & \alpha_{1}(t) L_{2} & \alpha_{1}^{2}(t) L_{1}\left(2 R_{1}\right)^{-1} L_{1}^{T}+\alpha_{1}^{2}(t) L_{2}\left(4 R_{1}\right)^{-1} L_{2}^{T}
\end{array}\right]\left[\begin{array}{l}
F_{1} \\
F_{2}
\end{array}\right], \\
& 2 \int_{t-\alpha(t)}^{t-\alpha_{1}} \int_{\theta}^{t-\alpha_{1}}\left[\begin{array}{c}
g \\
\lambda_{1} g
\end{array}\right]^{T}\left[\begin{array}{l}
X_{1} \\
X_{2}
\end{array}\right] \dot{w}(s) \mathrm{d} s \mathrm{~d} \theta=-\alpha_{1}(t)\left[\begin{array}{c}
F_{1} \\
F_{2}
\end{array}\right]^{T}\left[\begin{array}{ccc}
\frac{4}{\alpha_{1}(t)} R_{1} & 0 & L_{1}^{T} \\
0 & \frac{8}{\alpha_{1}(t)} R_{1} & L_{2}^{T} \\
L_{1} & L_{2} & 0
\end{array}\right]\left[\begin{array}{l}
F_{1} \\
F_{2}
\end{array}\right] \\
& =-\left[\begin{array}{l}
F_{1} \\
F_{2}
\end{array}\right]^{T}\left[\begin{array}{cc}
2 \widetilde{R}_{1} & \alpha_{1}(t) S_{1} \\
* & 0
\end{array}\right]\left[\begin{array}{l}
F_{1} \\
F_{2}
\end{array}\right], \\
& 2 \int_{t-\alpha_{2}}^{t-\alpha(t)} \int_{\theta}^{t-\alpha(t)}\left[\begin{array}{c}
g \\
\lambda_{1} g
\end{array}\right]^{T}\left[\begin{array}{cc}
X_{3} R_{2}^{-1} X_{3}^{-1} & X_{3} R_{2}^{-1} X_{4}^{-1} \\
& X_{4} R_{2}^{-1} X_{4}^{-1}
\end{array}\right]\left[\begin{array}{c}
g \\
\lambda_{2} g
\end{array}\right] \mathrm{d} s \mathrm{~d} \theta \\
& =\left[\begin{array}{l}
F_{1} \\
F_{2}
\end{array}\right]^{T}\left[\begin{array}{ccc}
\alpha_{2}^{2}(t) L_{3}\left(2 R_{2}\right)^{-1} L_{3}^{T}+\alpha_{2}^{2}(t) L_{4}\left(4 R_{2}\right)^{-1} L_{4}^{T} & \alpha_{2}(t) L_{3} & \alpha_{2}(t) L_{4} \\
\alpha_{2}(t) L_{3}^{T} & 2 R_{2} & 0 \\
\alpha_{2}(t) L_{4}^{T} & 0 & 4 R_{2}
\end{array}\right] \\
& =\left[\begin{array}{l}
F_{1} \\
F_{2}
\end{array}\right]^{T}\left[\begin{array}{lc}
\alpha_{2}^{2}(t) S_{2} \widetilde{R}_{2}^{-1} S_{2}^{T} & \alpha_{2}(t) S_{2} \\
\widetilde{R}_{2}
\end{array}\right]\left[\begin{array}{l}
F_{1} \\
F_{2}
\end{array}\right] \text {, } \\
& 2 \int_{t-\alpha_{2}}^{t-\alpha(t)} \int_{\theta}^{t-\alpha(t)}\left[\begin{array}{c}
g \\
\lambda_{2} g
\end{array}\right]\left[\begin{array}{l}
X_{3} \\
X_{4}
\end{array}\right] \dot{w}(s) \mathrm{d} s \mathrm{~d} \theta=-\alpha_{2}(t)\left[\begin{array}{l}
F_{1} \\
F_{2}
\end{array}\right]^{T}\left[\begin{array}{ccc}
0 & L_{3} & L_{4} \\
L_{3}^{T} \frac{4}{\alpha_{2}(t)} R_{2} & 0 \\
L_{4}^{T} & 0 & \frac{8}{\alpha_{2}(t)} R_{2}
\end{array}\right]\left[\begin{array}{l}
F_{1} \\
F_{2}
\end{array}\right] \\
& =-\left[\begin{array}{l}
F_{1} \\
F_{2}
\end{array}\right]^{T}\left[\begin{array}{cc}
0 & \alpha_{2}(t) S_{2} \\
2 & \widetilde{R}_{2}
\end{array}\right]\left[\begin{array}{l}
F_{1} \\
F_{2}
\end{array}\right] .
\end{aligned}
$$


Thus,

$$
\begin{aligned}
\Pi_{1}+\Pi_{2} \geq & {\left[\begin{array}{l}
F_{1} \\
F_{2}
\end{array}\right]^{T}\left\{\left[\begin{array}{cc}
\widetilde{R}_{1} & 0 \\
0 & \widetilde{R}_{2}
\end{array}\right]\right.} \\
& \left.-\left[\begin{array}{cc}
\alpha_{2}^{2}(t) S_{2} \widetilde{R}_{2}^{-1} S_{2}^{T} & 0 \\
0 & \alpha_{1}^{2}(t) S_{1}^{T} \widetilde{R}_{1}^{-1} S_{1}
\end{array}\right]\right\}\left[\begin{array}{l}
F_{1} \\
F_{2}
\end{array}\right] .
\end{aligned}
$$

This completes the proof of inequality.

Remark 4. From Lemma 3, the following double integral inequality can be written as

$$
\begin{gathered}
\int_{t-\alpha(t)}^{t-\alpha_{1}} \int_{\theta}^{t-\alpha_{1}} \dot{w}(s) R_{1} \dot{w}(s) \mathrm{d} s \mathrm{~d} \theta+\int_{t-\alpha_{2}}^{t-\alpha(t)} \int_{\theta}^{t-\alpha(t)} \\
\dot{w}^{T}(s) R_{2} \dot{w}(s) \mathrm{d} s \mathrm{~d} \theta \geq\left[\begin{array}{l}
F_{1} \\
F_{2}
\end{array}\right]^{T}\left[\begin{array}{cc}
\widetilde{R}_{1} & 0 \\
0 & \widetilde{R}_{2}
\end{array}\right]\left[\begin{array}{l}
F_{1} \\
F_{2}
\end{array}\right] .
\end{gathered}
$$
shown:

Compared with inequality (32), two aspects can be

(1) If $S_{1}=0$ and $S_{2}=0$, then (32) is reduced to (40) which it is a more general form. Based on Schur complement, (32) can be written as

$$
\left[\begin{array}{cccc}
\widetilde{R}_{1} & 0 & \alpha_{2}(t) F_{1}^{T} S_{2} & \alpha_{1}(t) F_{2}^{T} S_{1}^{T} \\
* & \widetilde{R}_{2} & 0 & 0 \\
* & * & \widetilde{R}_{2} & 0 \\
* & * & * & \widetilde{R}_{1}
\end{array}\right]>0
$$

(i) It provides the freedom matrices $S_{1}$ and $S_{2}$, which lead to reduced conservativeness.

(2) The right-hand side of (32) has information of nonlinear delayed terms $\alpha_{1}^{2}(t)$ and $\alpha_{2}^{2}(t)$. Time-delay terms appear in this term. It is the advantage to deal with a larger time delay system which can help to reduce conservatism.

\section{Novel Lyapunov Functionals}

In this section, we propose two novel Lyapunov functionals, which are the main contributions of this paper.

Proposition 1. For system (10) with given scalars $\sigma_{1}, \sigma_{2}, \tau_{1}$, and $\tau_{2}$ and positive definite matrices $M_{i}$ and $N_{i}$, $i=1,2,3,4$, the following function can be Lyapunov functional candidate:

$$
V_{J}(t)=V_{J 1}(t)+V_{J 2}(t)
$$

where

$$
\begin{aligned}
V_{J 1}(t)= & \tau_{1}(t) \int_{t-\tau(t)}^{t-\tau_{1}} \dot{x}^{T}(s) M_{1} \dot{x}(s) \mathrm{d} s+\tau_{2}(t) \int_{t-\tau_{2}}^{t-\tau(t)} \dot{x}^{T}(s) M_{2} \dot{x}(s) \mathrm{d} s \\
& +\sigma_{1}(t) \int_{t-\sigma(t)}^{t-\sigma_{1}} \dot{y}^{T}(s) N_{1} \dot{y}(s) \mathrm{d} s
\end{aligned}
$$$$
+\sigma_{2}(t) \int_{t-\sigma_{2}}^{t-\sigma(t)} \dot{y}^{T}(s) N_{2} \dot{y}(s) d s-\tau_{1}
$$$$
-\left(x\left(t-\tau_{1}\right)-x(t-\tau(t))\right)^{T} M_{1}(x(t-x(t-\tau(t))))
$$$$
-\left(x(t-\tau(t))-x\left(t-\tau_{2}\right)\right)^{T} M_{2}\left(x(t-\tau(t))-x\left(t-\tau_{2}\right)\right)
$$$$
-\left(y\left(t-\sigma_{1}\right)-y(t-\sigma(t))\right)^{T} N_{1}\left(y\left(t-\sigma_{1}\right)-y(t-\sigma(t))\right)
$$$$
-\left(y(t-\sigma(t))-y\left(t-\sigma_{2}\right)\right)^{T} N_{2}\left(y(t-\sigma(t))-y\left(t-\sigma_{2}\right)\right),
$$

$V_{J 2}(t)=\tau_{1}(t)\left(\int_{t-\tau(t)}^{t-\tau_{1}} \int_{\theta}^{t-\tau_{1}} \dot{x}^{T}(s) M_{3} \dot{x}(s) \mathrm{d} s \mathrm{~d} \theta-\eta_{1}^{T}(t) M_{3} \eta_{1}(t)\right)$

$$
\begin{aligned}
& +\tau_{2}(t)\left(\int_{t-\tau_{2}}^{t-\tau(t)} \int_{\theta}^{t-\tau(t)} \dot{x}^{T}(s) M_{4} \dot{x}(s) \mathrm{d} s \mathrm{~d} \theta-\eta_{2}(t) M_{4} \eta_{2}(t)\right) \\
& +\sigma_{1}(t)\left(\int_{t-\sigma(t)}^{t-\sigma_{1}} \int_{\theta}^{t-\sigma_{1}} \dot{y}^{T}(s) N_{3} \dot{y}(s) \mathrm{d} s \mathrm{~d} \theta-\eta_{3}^{T}(t) N_{3} \eta_{3}(t)\right)
\end{aligned}
$$

$$
+\sigma_{2}(t)\left(\int_{t-\sigma_{2}}^{t-\sigma(t)} \int_{\theta}^{t-\sigma(t)} \dot{y}^{T}(s) N_{4} \dot{y}(s) \mathrm{d} s \mathrm{~d} \theta-\eta_{4}^{T}(t) N_{4} \eta_{4}(t)\right)
$$

$\eta_{1}(t)=x\left(t-\tau_{1}\right)-\int_{t-\tau(t)}^{t-\tau_{1}} \frac{x(s)}{\tau_{1}(t)} \mathrm{d} s$

$$
\eta_{2}(t)=x(t-\tau(t))-\int_{t-\tau(t)}^{t-\tau_{2}} \frac{x(s)}{\tau_{2}(t)} \mathrm{d} s
$$

$$
\eta_{3}(t)=y\left(t-\sigma_{1}\right)-\int_{t-\sigma(t)}^{t-\sigma_{1}} \frac{y(s)}{\sigma_{1}(t)} \mathrm{d} s
$$

$$
\eta_{4}(t)=y(t-\sigma(t))-\int_{t-\sigma_{2}}^{t-\sigma(t)} \frac{y(s)}{\sigma_{2}(t)} \mathrm{d} s
$$


Proof. By Lemma $1, V_{J}(t)$ is a positive definite function which completes the proof.

Remark 5. The construction of Lyapunov functionals is usually in the form of ALFA and MILFA. The special formed function (42) in Proposition 1 is based on Jensen integral inequality and relax double integral inequality, respectively. It is obtained in the following points:

(i) The proposed functions are the product of the timevarying delays; the time-varying delays and rate of the change of the time-varying delays are associated with $\tau(t), \sigma(t), \dot{\tau}(t)$, and $\dot{\sigma}(t)$. Thus, the proposed functional may be of some advantage to find the stability conditions for genetic regulatory networks with interval time-varying delays.

(ii) There are nonpositive definite terms, i.e., $-(\mathrm{x}(\mathrm{t}-$ $\left.\left.\tau_{1}\right)-x(t-\tau(t))\right)^{T} M_{1}\left(x\left(t-\tau_{1}\right)-x(t-\tau(t))\right),-(x$ $\left.(t-\tau(t))-x\left(t-\tau_{2}\right)\right)^{T} M_{2}\left(x(t-\tau(t))-x\left(t-\tau_{2}\right)\right)$, $-\left(\mathrm{y}\left(t-\sigma_{1}\right)-y(t-\sigma(t))\right)^{T} N_{1}\left(y\left(t-\sigma_{1}\right)-y(t-\sigma\right.$ $(t)))$, and $-\left(y(t-\sigma(t))-y\left(t-\sigma_{2}\right)\right)^{T} N_{2}(y(t-\sigma$ $\left.(t))-y\left(t-\sigma_{2}\right)\right)$ in $V_{J 1}(t)$ and $-\tau_{1}(t) \eta_{1}^{T}(t) M_{3} \eta_{1}(t)$, $-\tau_{2}(t) \eta_{2}^{T}(t) M_{4} \eta_{2}(t), \quad-\sigma_{1}(t) \eta_{3}^{T}(t) N_{3} \eta_{3}(t), \quad$ and $-\sigma_{2}(t) \eta_{4}^{T}(t) N_{4} \eta_{4}(t)$ in $V_{J 2}(t)$. These terms would play an important role in relaxing the stability condition.

Remark 6. The new Lyapunov functional has different forms compared with $[32,33]$, i.e., there are two double integral terms, $\quad \tau_{1}(t) \int_{t-\tau(t)}^{t-\tau_{1}} \int_{\theta}^{t-\tau_{1}} \dot{x}^{T}(s) M_{3} \dot{x}(s) \mathrm{d} s \mathrm{~d} \theta, \quad \tau_{2}(t) \int_{t-\tau_{2}}^{t-\tau(t)}$ $\int_{\theta}^{t-\tau(t)} \dot{x}^{T}(s) M_{4} \dot{x}(s) \mathrm{d} s \mathrm{~d} \theta, \quad \sigma_{1}(t) \int_{t-\sigma(t)}^{t-\sigma_{1}} \int_{\theta}^{t-\sigma_{1}} \dot{y}^{T}(s) N_{3}$ $\dot{y}(s) \mathrm{d} s \mathrm{~d} \theta$, and $\sigma_{2}(t) \int_{t-\sigma_{2}}^{t-\sigma(t)} \int_{\theta}^{t-\sigma(t)} \dot{y}^{T}(s) N_{4} \dot{y}(s) \mathrm{d} s \mathrm{~d} \theta$. These terms are increasing the information in stability conditions which can help in delay conditions for GRNs with interval time-varying delays.

\section{Main Results}

In this section, we analyse the asymptotic stability of genetic regulatory networks (10) with time-varying delays. The main theorem given below shows that the stability criteria can be expressed in the terms of the feasibility of the linear matrix inequalities (LMIs).

For simplification, the following vectors and matrices are defined for later use:

$$
\begin{aligned}
& \xi(t)=\operatorname{col}\left\{\begin{array}{c}
x(t), y(t), x\left(t-\tau_{1}\right), x\left(t-\tau_{2}\right), x(t-\tau(t)), y\left(t-\sigma_{1}\right), y\left(t-\sigma_{2}\right), y(t-\sigma(t)) \\
f(y(t)), f(y(t-\sigma(t))), \int_{t-\tau_{1}}^{t} \frac{x(s)}{\tau_{1}} \mathrm{~d} s, \int_{t-\tau(t)}^{t-\tau_{1}} \frac{x(s)}{\tau_{1}(t)} \mathrm{d} s, \int_{t-\tau_{2}}^{t-\tau(t)} \frac{x(s)}{\tau_{2}(t)} \mathrm{d} s, \int_{t-\sigma_{1}}^{t} \frac{y(s)}{\sigma_{1}} \mathrm{~d} s, \\
\int_{t-\sigma(t)}^{t-\sigma_{1}} \frac{y(s)}{\sigma_{1}(t)} \mathrm{d} s, \int_{t-\sigma_{2}}^{t-\sigma(t)} \frac{y(s)}{\sigma_{2}(t)} \mathrm{d} s, \int_{t-\tau_{1}}^{t} \int_{\theta}^{t} \frac{x(s)}{\tau_{1}^{2}} \mathrm{~d} s \mathrm{~d} \theta, \int_{t-\tau(t)}^{t-\tau_{1}} \int_{\theta}^{t} \frac{x(s)}{\tau_{1}^{2}(t)} \mathrm{d} s \mathrm{~d} \theta, \\
\int_{t-\tau_{2}}^{t-\tau(t)} \int_{\theta}^{t} \frac{x(s)}{\tau_{2}^{2}(t)} \mathrm{d} s \mathrm{~d} \theta, \int_{t-\sigma_{1}}^{t} \int_{\theta}^{t} \frac{y(s)}{\sigma_{1}^{2}} \mathrm{~d} s \mathrm{~d} \theta, \int_{t-\sigma(t)}^{t-\sigma_{1}} \int_{\theta}^{t} \frac{y(s)}{\sigma_{1}^{2}(t)} \mathrm{d} s \mathrm{~d} \theta, \int_{t-\sigma_{2}}^{t-\sigma(t)} \int_{\theta}^{t} \frac{y(u)}{\sigma_{2}^{2}(t)} \mathrm{d} s \mathrm{~d} \theta, \\
x\left(t-\tau_{1}\right), \dot{x}\left(t-\tau_{2}\right), \dot{x}(t-\tau(t)), \dot{y}\left(t-\sigma_{1}\right), \dot{y}\left(t-\sigma_{2}\right), \dot{y}(t-\sigma(t))
\end{array},\right. \\
& \tau_{21}=\tau_{2}-\tau_{1}, \\
& \tau_{1}(t)=\tau(t)-\tau_{1}, \\
& \tau_{2}(t)=\tau_{2}-\tau(t), \\
& \dot{\tau}_{d}=1-\dot{\tau}(t), \\
& \sigma_{21}=\sigma_{2}-\sigma_{1}, \\
& \sigma_{1}(t)=\sigma(t)-\sigma_{1}, \\
& \sigma_{2}(t)=\sigma_{2}-\sigma(t),
\end{aligned}
$$




$$
\begin{aligned}
& \dot{\sigma}_{d}=1-\dot{\sigma}(t), \\
& \eta_{1}(t)=e_{2}-e_{12} \text {, } \\
& \eta_{2}(t)=e_{5}-e_{13}, \\
& \eta_{3}(t)=e_{6}-e_{15}, \\
& \eta_{4}(t)=e_{8}-e_{16} \text {, } \\
& \psi_{1}=\left[\begin{array}{c}
e_{1}-e_{3} \\
e_{1}+e_{3}-2 e_{11} \\
e_{1}-e_{3}+6 e_{11}-12 e_{17}
\end{array}\right], \\
& \psi_{2}=\left[\begin{array}{c}
e_{2}-e_{6} \\
e_{2}+e_{6}-2 e_{24} \\
e_{2}-e_{6}+6 e_{24}-12 e_{20}
\end{array}\right] \text {, } \\
& \psi_{3}=\left[\begin{array}{c}
e_{3}-e_{5} \\
e_{3}+e_{5}-2 e_{12} \\
e_{3}-e_{5}+6 e_{12}-12 e_{18}
\end{array}\right] \text {, } \\
& \psi_{4}=\left[\begin{array}{c}
e_{6}-e_{8} \\
e_{6}+e_{8}-2 e_{15} \\
e_{6}-e_{8}+6 e_{15}-12 e_{21}
\end{array}\right] \text {, } \\
& \psi_{5}=\left[\begin{array}{c}
e_{1}-e_{11} \\
e_{1}+2 e_{11}-6 e_{17}
\end{array}\right] \text {, } \\
& \psi_{6}=\left[\begin{array}{c}
e_{2}-e_{14} \\
e_{2}+2 e_{14}-6 e_{20}
\end{array}\right] \text {, } \\
& \psi_{7}=\left[\begin{array}{c}
e_{5}-e_{4} \\
e_{5}+e_{4}-2 e_{13} \\
e_{5}-e_{4}+6 e_{13}-12 e_{18}
\end{array}\right] \text {, } \\
& \psi_{8}=\left[\begin{array}{c}
e_{8}-e_{7} \\
e_{8}+e_{7}-2 e_{16} \\
e_{8}-e_{7}+6 e_{16}-12 e_{22}
\end{array}\right] \text {, } \\
& \pi_{1}=\left[\begin{array}{l}
E_{1} \\
E_{2}
\end{array}\right] \\
& \pi_{2}=\left[\begin{array}{l}
E_{3} \\
E_{4}
\end{array}\right] \\
& \pi_{3}=\left[\begin{array}{l}
F_{1} \\
F_{2}
\end{array}\right] \\
& \pi_{4}=\left[\begin{array}{l}
F_{3} \\
F_{4}
\end{array}\right]
\end{aligned}
$$

And $e_{i} \in \mathbb{R}^{n \times 28 n} \quad$ is defined as $e_{i}=$ $\left[\begin{array}{lll}0_{n \times(i-1) n} & I_{n} & 0_{n \times(28-i) n}\end{array}\right]$ for $i=1,2, \ldots, 28$.

Theorem 1. For given scalars $\sigma_{1}, \sigma_{2}, \tau_{1}, \tau_{2}, \sigma_{d}$, and $\tau_{d}$, the genetic regulatory networks (10) with time-varying delays subject to assumptions 1 and 2 which are asymptotically stable, if there exist matrices $P \in \mathbb{S}_{+}^{n \times n}, \quad R_{i} \in \mathbb{S}_{+}^{n \times n}$ $(i=1,2,3,4,6), R_{5} \in \mathbb{S}_{+}^{2 n \times 2 n}, W_{i}, X_{i} \in \mathbb{S}_{+}^{n \times n}(i=1,2,3,4)$, and $M_{i}, N_{i} \in \mathbb{S}_{+}^{n \times n}(i=1,2,3,4)$, diagonal matrices $\Lambda_{i}, \in \mathbb{S}_{+}^{n \times n}$ and $H_{k} \in \mathbb{S}_{+}^{n \times n}(i=1,2)$, and any matrices $S_{i}, T_{i} \in \mathbb{R}^{2 n \times 2 n}(i=1,2,3,4)$ such that the following linear matrix inequalities (LMIs) hold: 


$$
\begin{aligned}
& W_{2}-\dot{\tau}(t) M_{1}>0, W_{2}+\dot{\tau}(t) M_{2}>0, \\
& W_{4}-\dot{\sigma}(t) N_{1}>0, W_{4}+\dot{\sigma}(t) N_{2}>0, \\
& X_{2}-\dot{\tau}(t) M_{3}>0, X_{2}+\dot{\tau}(t) M_{4}>0, \\
& X_{4}-\dot{\sigma}(t) N_{3}>0, X_{4}+\dot{\sigma}(t) N_{4}>0,
\end{aligned}
$$

$$
\left[\begin{array}{ccc}
\Sigma_{1} & E_{1}^{T} S_{2} & \tau_{21} F_{1}^{T} T_{2} \\
* & -\tau_{21} \overline{\mathscr{W}}_{2} & 0 \\
* & * & -\overline{\mathscr{X}}_{2}
\end{array}\right]<0,\left[\begin{array}{ccc}
\Sigma_{2} & E_{2}^{T} S_{1}^{T} & \tau_{21} F_{2}^{T} T_{1} \\
* & -\tau_{21} \overline{\mathscr{W}}_{1} & 0 \\
* & * & -\overline{\mathscr{X}}_{1}
\end{array}\right]<0,
$$

$$
\left[\begin{array}{ccc}
\Sigma_{3} & E_{3}^{T} S_{4} & \sigma_{21} F_{3}^{T} T_{4} \\
* & -\sigma_{21} \overline{\mathscr{W}}_{4} & 0 \\
* & * & -\overline{\mathscr{X}}_{4}
\end{array}\right]<0,\left[\begin{array}{ccc}
\Sigma_{4} & E_{4}^{T} S_{3}^{T} & \sigma_{21} F_{4}^{T} T_{3} \\
* & -\sigma_{21} \overline{\mathscr{W}}_{3} & 0 \\
* & * & -\overline{\mathscr{X}}_{3}
\end{array}\right]<0,
$$

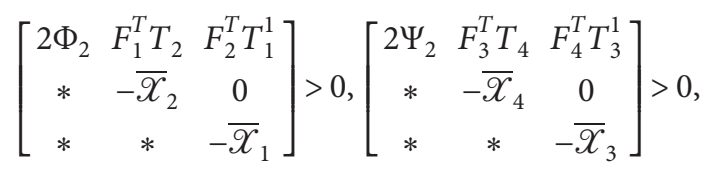

where

$$
\begin{aligned}
\Sigma_{1}= & \tau_{1}^{2} \Phi_{2}+\tau_{1} \Phi_{1}+\Phi_{0}+\Omega_{x}(\dot{\tau}(t)) \\
& -\frac{1}{\tau_{21}} \pi_{1}^{T}\left[\begin{array}{cc}
2 \overline{\mathscr{W}}_{1} & S_{1} \\
* & \overline{\mathscr{W}}_{2}
\end{array}\right] \pi_{1}-\pi_{3}^{T}\left[\begin{array}{cc}
\overline{\mathscr{X}}_{1} & 0 \\
0 & \overline{\mathscr{X}}_{2}
\end{array}\right] \pi_{3}, \\
\Sigma_{2}= & \tau_{2}^{2} \Phi_{2}+\tau_{2} \Phi_{1}+\Phi_{0}+\Omega_{x}(\dot{\tau}(t))-\frac{1}{\tau_{21}} \pi_{1}^{T}\left[\begin{array}{cc}
\overline{\mathscr{W}}_{1} & S_{2} \\
* & 2 \overline{\mathscr{W}}_{2}
\end{array}\right] \pi_{1} \\
& -\pi_{3}^{T}\left[\begin{array}{cc}
\bar{X}_{1} & 0 \\
0 & \overline{\mathscr{X}}_{2}
\end{array}\right] \pi_{3},
\end{aligned}
$$

$\Sigma_{3}=\sigma_{1}^{2} \Psi_{2}+\sigma_{1} \Psi_{1}+\Psi_{0}+\Omega_{y}(\dot{\sigma}(t))-\frac{1}{\sigma_{21}} \pi_{2}^{T}\left[\begin{array}{cc}2 \overline{\mathscr{W}}_{3} & S_{3} \\ * & \overline{\mathscr{W}}_{4}\end{array}\right] \pi_{2}$ $-\pi_{4}^{T}\left[\begin{array}{cc}\bar{X}_{3} & 0 \\ 0 & \mathscr{X}_{4}\end{array}\right] \pi_{4}$

$$
\begin{aligned}
\Sigma_{4}= & \sigma_{2}^{2} \Psi_{2}+\sigma_{2} \Psi_{1}+\Psi_{0}+\Omega_{y}(\dot{\sigma}(t))-\frac{1}{\sigma_{21}} \pi_{2}^{T}\left[\begin{array}{cc}
\overline{\mathscr{W}}_{3} & S_{4} \\
* & 2 \overline{\mathscr{W}}_{4}
\end{array}\right] \pi_{2} \\
& -\pi_{4}^{T}\left[\begin{array}{cc}
\overline{\mathscr{X}}_{3} & 0 \\
0 & \mathscr{X}_{4}
\end{array}\right] \pi_{4},
\end{aligned}
$$

$$
\Omega(\dot{\tau}(t), \dot{\sigma}(t))=\Omega_{x}(\dot{\tau}(t))+\Omega_{y}(\dot{\sigma}(t)),
$$

$$
\begin{aligned}
\Omega_{x}(\dot{\tau}(t))= & e_{1}^{T} P e_{1}+e_{1}^{T}\left(R_{1}+R_{2}\right) e_{1}+e_{3}^{T}\left(R_{3}-R_{1}\right) e_{3} \\
& -\tau_{d} e_{5}^{T} R_{2} e_{5}-e_{4}^{T} R_{3} e_{4} \\
& +\left(-A e_{1}+W e_{10}\right)^{T}\left(\tau_{1} W_{1}+\tau_{21} W_{2}\right)\left(-A e_{1}+W e_{10}\right) \\
& +\left(-A e_{1}+W e_{10}\right)^{T} \\
& \times\left(\frac{\tau_{1}^{2}}{2} X_{1}+\frac{\tau_{21}^{2}}{2} X_{2}\right)\left(-A e_{1}+W e_{10}\right)+\psi_{1}^{T} \widetilde{W}_{1} \psi_{1} \\
& +\psi_{3}^{T}\left(\widetilde{X}_{2}+\tilde{M}_{3}\right) \psi_{3}+\psi_{5}^{T} \widetilde{X}_{1} \psi_{5}+\psi_{7}^{T} \tilde{M}_{4} \psi_{7}
\end{aligned}
$$

$$
\begin{aligned}
\Omega_{y}(\dot{\tau}(t))= & \operatorname{Sym}\left\{\left(L e_{2}-e_{9}\right)^{T} \Lambda_{1}\left(-C e_{2}+D e_{5}\right)\right. \\
& \left.+e_{9}^{T} \Lambda_{2}\left(-C e_{2}+D e_{5}\right)\right\}+e_{1}^{T} P e_{1}+e_{2}^{T} R_{4} e_{2} \\
& +\left[\begin{array}{c}
e_{2} \\
e_{9}
\end{array}\right]^{T} R_{5}\left[\begin{array}{c}
e_{2} \\
e_{9}
\end{array}\right]^{T}+e_{6}^{T} R_{6} e_{6} \\
& -\sigma_{d}\left[\begin{array}{c}
e_{8} \\
e_{10}
\end{array}\right]^{T} R_{5}\left[\begin{array}{c}
e_{8} \\
e_{10}
\end{array}\right] \\
& -e_{7}^{T} R_{6} e_{7}+\left(-C e_{2}+D e_{5}\right)^{T} \\
& \times\left(\sigma_{1} W_{3}+\sigma_{21} W_{4}\right)\left(-C e_{2}+D e_{5}\right) \\
& +\left(-C e_{2}+D e_{5}\right)^{T}\left(\frac{\sigma_{1}^{2}}{2} X_{3}+\frac{\sigma_{21}^{2}}{2} X_{4}\right) \\
& \times\left(-C e_{2}+D e_{5}\right) \\
& +\psi_{2}^{T} \widetilde{W}_{3} \psi_{2}+\psi_{4}^{T}\left(\widetilde{X}_{4}+\widetilde{N}_{3}\right) \psi_{4}+\psi_{6}^{T} \widetilde{X}_{3} \psi_{6} \\
& +\psi_{8}^{T} \tilde{N}_{4} \psi_{8} \\
& -\operatorname{Sym}\left\{\left(e_{9}-L e_{2}\right)^{T} H_{1}+\left(e_{10}-L e_{8}\right)^{T} H_{2} e_{10}\right\}
\end{aligned}
$$

$$
\begin{aligned}
& \Omega_{1}(\tau(t), \dot{\tau}(t))=\Phi_{2} \tau^{2}(t)+\Phi_{1} \tau(t)+\Phi_{0}, \\
& \Phi_{2}=e_{23}^{T} M_{3} e_{23}+\tau_{d} e_{25}^{T} M_{4} e_{25} \text {, } \\
& \Phi_{1}=e_{23}^{T}\left(M_{1}-2 \tau_{1} M_{3}\right) e_{23}-\tau_{d} e_{25}^{T}\left(M_{1}+M_{2}+2 \tau_{2} M_{4}\right) e_{25} \\
& +e_{24}^{T} M_{2} e_{24} \\
& -\operatorname{Sym}\left\{\eta_{1}^{T}(t) M_{3} e_{23}-\tau_{d} \eta_{2}^{T}(t) M_{4} e_{25}\right\}, \\
& \Phi_{0}=e_{23}^{T}\left(\tau_{1}^{2} M_{3}+\tau_{1} M_{1}\right) e_{23} \\
& +\tau_{d} e_{25}^{T}\left(\tau_{2}^{2} M_{4}+\tau_{2} M_{2}+\tau_{1} M_{1}\right) e_{25}-\tau_{2} e_{24}^{T} M_{2} e_{24} \\
& +\operatorname{Sym}\left\{\tau_{1} \eta_{1}^{T}(t) M_{3} e_{23}-\tau_{2} \tau_{d} \eta_{2}^{T}(t) M_{4} e_{25}\right\} \\
& -\operatorname{Sym}\left\{\left(e_{3}-e_{5}\right)^{T} M_{1}\left(e_{23}-\tau_{d} e_{25}\right)\right. \\
& \left.+\left(e_{5}-e_{4}\right)^{T} M_{2}\left(\tau_{d} e_{25}-e_{24}\right)\right\} \\
& -\frac{\dot{\tau}(t)}{\tau_{21}^{2}}\left(\eta_{1}^{T}(t) M_{3} \eta_{1}(t)-\eta_{2}^{T}(t) M_{4} \eta_{2}(t)\right) \\
& +\operatorname{Sym}\left\{\begin{array}{c}
\eta_{1}^{T}(t) M_{3}\left(e_{3}-\tau_{d} e_{5}+\dot{\tau}(t) e_{12}\right) \\
+\eta_{2}^{T}(t) M_{4}\left(\tau_{d} e_{5}-e_{4}+\dot{\tau}(t) e_{13}\right)
\end{array}\right\},
\end{aligned}
$$




$$
\begin{aligned}
\Omega_{2} & (\sigma(t), \dot{\sigma}(t))=\Psi_{2} \sigma^{2}(t)+\Psi_{1} \sigma(t)+\Psi_{0}, \\
\Psi_{2}= & e_{26}^{T} N_{3} e_{26}+\sigma_{d} e_{28}^{T} N_{4} e_{26}, \\
\Psi_{1}= & e_{26}^{T}\left(N_{1}-2 \sigma_{1} N_{3}\right) e_{26} \\
& -\sigma_{d} e_{28}^{T}\left(N_{1}+N_{2}+2 \sigma_{2} N_{4}\right) e_{28}+e_{27}^{T} N_{2} e_{27} \\
& -\operatorname{Sym}\left\{\eta_{3}^{T}(t) N_{3} e_{26}-\sigma_{d} \eta_{4}^{T}(t) N_{4} e_{28}\right\}, \\
\Phi_{0}= & e_{26}^{T}\left(\sigma_{1}^{2} N_{3}+\sigma_{1} N_{1}\right) e_{26} \\
& +\tau_{d} e_{25}^{T}\left(\sigma_{2}^{2} N_{4}+\sigma_{2} N_{2}+\sigma_{1} N_{1}\right) e_{25}-\sigma_{2} e_{27}^{T} N_{2} e_{27} \\
& +\operatorname{Sym}_{2}\left\{\sigma_{1} \eta_{3}^{T}(t) N_{3} e_{26}-\sigma_{2} \sigma_{d} \eta_{4}^{T}(t) N_{4} e_{26}\right\} \\
& \left.-\operatorname{Sym}\left\{e_{6}-e_{8}\right)^{T} N_{1}\left(e_{26}-\sigma_{d} e_{28}\right)\right\} \\
& \left.+\left(e_{8}-e_{7}\right)^{T} N_{2}\left(\sigma_{d} e_{28}-e_{27}\right)\right\} \\
& -\frac{\sigma^{2}(t)}{\sigma_{21}^{2}}\left(\eta_{3}^{T}(t) N_{3} \eta_{3}(t) \eta_{4}^{T}(t) N_{4} \eta_{4}(t)\right) \\
& +\operatorname{Sym}\left\{\eta_{3}^{T}(t) N_{3}\left(e_{6}-\sigma_{d} e_{8}+\dot{\tau}(t) e_{15}\right)\right. \\
& \left.+\eta_{4}^{T}(t) N_{4}\left(\sigma_{d} e_{8}-e_{7}+\dot{\tau}(t) e_{16}\right)\right\},
\end{aligned}
$$

$\overline{\mathscr{W}}_{1}=\operatorname{diag}\left\{W_{2}-\dot{\tau}(t) M_{1}, 3\left(W_{2}-\dot{\tau}(t) M_{1}\right)\right\}$,

$\overline{\mathscr{W}}_{2}=\operatorname{diag}\left\{W_{2}+\dot{\tau}(t) M_{2}, 3\left(W_{2}+\dot{\tau}(t) M_{2}\right)\right\}$,

$\overline{\mathscr{W}}_{3}=\operatorname{diag}\left\{W_{4}-\dot{\sigma}(t) N_{1}, 3\left(W_{4}-\dot{\sigma}(t) N_{1}\right)\right\}$,

$\overline{\mathscr{W}}_{4}=\operatorname{diag}\left\{W_{4}+\dot{\sigma}(t) N_{2}, 3\left(W_{4}+\dot{\sigma}(t) N_{2}\right)\right\}$,

$\overline{\mathscr{X}}_{1}=\operatorname{diag}\left\{2\left(X_{2}-\dot{\tau}(t) M_{3}\right), 4\left(W_{2}-\dot{\tau}(t) M_{3}\right)\right\}$,

$\bar{X}_{2}=\operatorname{diag}\left\{2\left(X_{2}+\dot{\tau}(t) M_{4}\right), 4\left(X_{2}+\dot{\tau}(t) M_{4}\right)\right\}$,

$\bar{X}_{3}=\operatorname{diag}\left\{2\left(X_{4}-\dot{\sigma}(t) N_{3}\right), 4\left(X_{4}-\dot{\sigma}(t) N_{3}\right)\right\}$,

$\bar{X}_{4}=\operatorname{diag}\left\{2\left(X_{4}+\dot{\sigma}(t) N_{4}\right), 4\left(X_{4}+\dot{\sigma}(t) N_{4}\right)\right\}$,

$\Pi_{5}=\operatorname{diag}\left\{\tilde{W}_{1}, \widetilde{W}_{3}, \tilde{X}_{2}+\tilde{M}_{3}, \tilde{X}_{4}+\tilde{N}_{3}, \tilde{X}_{1}, \tilde{X}_{3}, \tilde{M}_{4}, \tilde{N}_{4}\right\}$,

$\widetilde{W}_{i}=\operatorname{diag}\left\{W_{i}, 3 W_{i}, 5 W_{i}\right\}$,

$\tilde{X}_{i+1}=\operatorname{diag}\left\{X_{i+1}, 3 X_{i+1}, 5 X_{i+1}\right\}$,

$\widetilde{X}_{i}=\operatorname{diag}\left\{2 X_{i}, 4 X_{i}\right\}$,

$\tilde{M}_{4}=\operatorname{diag}\left\{M_{4}, 3 M_{4}, 5 M_{4}\right\}$,

$\tilde{N}_{4}=\operatorname{diag}\left\{N_{4}, 3 N_{4}, 5 N_{4}\right\}, \quad i=1,3$,

$\psi=\operatorname{col}\left\{\psi_{1}, \psi_{2}, \psi_{3}, \psi_{4}, \psi_{5}, \psi_{6}, \psi_{7}, \psi_{8}\right\}$.

Proof. We first consider the following Lyapunov functional candidate:

$$
V_{1}(t)=V_{0}(t)+V_{J}(t)
$$

where $V_{0}(t)=\sum_{i=1}^{4} V_{i}(t)$ and $V_{J}(t)$ is defined in Proposition 1:

$$
\begin{aligned}
& V_{1}(t)=\left[\begin{array}{l}
x(t) \\
y(t)
\end{array}\right]^{T}\left[\begin{array}{ll}
P & 0 \\
0 & P
\end{array}\right]\left[\begin{array}{l}
x(t) \\
y(t)
\end{array}\right]-2 \sum_{i=1}^{n} \\
& \int_{0}^{y_{i}(t)}\left(\lambda_{1 i}\left(l_{i} s-f_{i}(s)+\lambda_{2 i} f_{i}(s)\right)\right) \mathrm{d} s, \\
& V_{2}(t)=\int_{t-\tau_{1}}^{t} x^{T}(s) R_{1} x(s) \mathrm{d} s+\int_{t-\tau(t)}^{t} x^{T}(s) R_{2} x(s) \mathrm{d} s \\
& +\int_{t-\tau_{2}}^{t-\tau_{1}} x^{T}(s) R_{3} x(s) \mathrm{d} s \\
& +\int_{t-\sigma_{1}}^{t} x^{T}(s) R_{4} y(s) \mathrm{d} s+\int_{t-\sigma(t)}^{t}\left[\begin{array}{c}
y(s) \\
f(y(s))
\end{array}\right]^{T} \\
& R_{5}\left[\begin{array}{c}
y(s) \\
f(y(s))
\end{array}\right] \mathrm{d} s+\int_{t-\sigma_{2}}^{t-\sigma_{1}} y^{T} R_{6} y(s) \mathrm{d} s, \\
& V_{3}(t)=\int_{t-\tau_{1}}^{t} \int_{\theta}^{t} \dot{x}^{T}(s) W_{1} \dot{x}(s) \mathrm{d} s \mathrm{~d} \theta \\
& +\int_{t-\tau_{2}}^{t-\tau_{1}} \int_{\theta}^{t} \dot{x}^{T}(s) W_{2} \dot{x}(s) \mathrm{d} s \mathrm{~d} \theta \\
& +\int_{t-\sigma_{1}}^{t} \int_{\theta}^{t} \dot{y}(s) W_{3} \dot{y}(s) \mathrm{d} s \mathrm{~d} \theta \\
& +\int_{t-\sigma_{2}}^{t-\sigma_{1}} \int_{\theta}^{t} \dot{y}(s) W_{4} \dot{y}(s) \mathrm{d} s \mathrm{~d} \theta, \\
& V_{4}(t)=\int_{t-\tau_{1}}^{t} \int_{u}^{t} \int_{\theta}^{t} \dot{x}(s) X_{1} \dot{x}(s) \mathrm{d} s \mathrm{~d} \theta \mathrm{d} u \\
& +\int_{t-\tau_{2}}^{t-\tau_{1}} \int_{u}^{t-\tau_{1}} \int_{\theta}^{t} \dot{x}(s) X_{2} \dot{x}(s) \mathrm{d} s \mathrm{~d} \theta \mathrm{d} u \\
& +\int_{t-\sigma_{1}}^{t} \int_{u}^{t} \int_{\theta}^{t} \dot{y}^{T}(s) X_{3} \dot{y}(s) \mathrm{d} s \mathrm{~d} \theta \mathrm{d} u \\
& +\int_{t-\sigma_{2}}^{t-\sigma_{1}} \int_{u}^{t-\sigma_{1}} \int_{\theta}^{t} \dot{y}^{T}(s) X_{4} \dot{y}(s) \mathrm{d} s \mathrm{~d} \theta \mathrm{d} u .
\end{aligned}
$$

Moreover, we consider the following simple calculation results:

$$
\begin{aligned}
\frac{\mathrm{d}}{\mathrm{d} t}\left(\tau_{1}(t) \eta_{1}^{T}(t) M_{3} \eta_{1}(t)\right)= & \dot{\tau}(t) \eta_{1}^{T}(t) M_{3} \eta_{1}(t) \\
& +\operatorname{Sym}\left\{\eta_{1}^{T}(t) M_{3} \eta_{5}(t)\right\}, \\
\frac{\mathrm{d}}{\mathrm{d} t}\left(\tau_{2}(t) \eta_{2}^{T}(t) M_{4} \eta_{2}(t)\right)= & -\dot{\tau}(t) \eta_{2}^{T}(t) M_{4} \eta_{2}(t) \\
& +\operatorname{Sym}\left\{\eta_{2}^{T}(t) M_{4} \eta_{6}(t)\right\}, \\
\frac{\mathrm{d}}{\mathrm{d} t}\left(\sigma_{1}(t) \eta_{3}^{T}(t) N_{3} \eta_{3}(t)\right)= & \dot{\sigma}(t) \eta_{3}^{T}(t) N_{3} \eta_{3}(t) \\
& +\operatorname{Sym}\left\{\eta_{3}^{T}(t) N_{3} \eta_{7}(t)\right\}, \\
\frac{\mathrm{d}}{\mathrm{d} t}\left(\sigma_{2}(t) \eta_{4}^{T}(t) N_{4} \eta_{4}(t)\right)= & -\dot{\sigma}(t) \eta_{4}^{T}(t) N_{4} \eta_{4}(t) \\
& +\operatorname{Sym}\left\{\eta_{4}^{T}(t) N_{4} \eta_{8}(t)\right\},
\end{aligned}
$$

where 


$$
\begin{aligned}
& \eta_{5}(t)=\tau_{1}(t) \dot{x}\left(t-\tau_{1}\right)-x\left(t-\tau_{1}\right)+\tau_{d} x(t-\tau(t))-\dot{\tau}(t) \int_{t-\tau(t)}^{t-\tau_{1}} \frac{x(s)}{\tau_{1}(t)} \mathrm{d} s, \\
& \eta_{6}(t)=\tau_{2}(t) \tau_{d}(t) \dot{x}(t-\tau(t))-\tau_{d} x(t-\tau(t))+x\left(t-\tau_{2}\right)-\dot{\tau}(t) \int_{t-\tau_{2}}^{t-\tau(t)} \frac{x(s)}{\tau_{2}(t)} \mathrm{d} s, \\
& \eta_{7}(t)=\sigma_{1}(t) \dot{y}\left(t-\sigma_{1}\right)-y\left(t-\sigma_{1}\right)+\sigma_{d} y(t-\sigma(t))-\dot{\sigma}(t) \int_{t-\sigma(t)}^{t-\sigma_{1}} \frac{y(s)}{\sigma_{1}(t)} \mathrm{d} s, \\
& \eta_{8}(t)=\sigma_{2}(t) \sigma_{d}(t) \dot{y}(t-\sigma(t))-\sigma_{d} y(t-\sigma(t))+y\left(t-\sigma_{2}\right)-\dot{\sigma}(t) \int_{t-\sigma_{2}}^{t-\sigma(t)} \frac{y(s)}{\sigma_{2}(t)} \mathrm{d} s .
\end{aligned}
$$

Then, taking time derivatives of $V(t)$ along the trajectory where of system (10) yields:

$$
\dot{V}_{0}(t)=\sum_{i=1}^{4} \dot{V}_{i}(t),
$$

$$
\begin{aligned}
& \dot{V}_{1}(t)=\left[\begin{array}{l}
x(t) \\
y(t)
\end{array}\right]^{T}\left[\begin{array}{ll}
P & 0 \\
0 & P
\end{array}\right]\left[\begin{array}{l}
\dot{x}(t) \\
\dot{y}(t)
\end{array}\right] \\
& +2(L y(t)-f(y(t)))^{T} \Lambda_{1} \dot{y}(t)+2 f^{T}(y(t)) \Lambda_{2} \dot{y}(t)=\xi^{T}(t) \operatorname{Sym}\left\{\Pi_{1}\right\} \xi(t), \\
& \dot{V}_{2}(t)=x^{T}(t)\left(R_{1}+R_{2}\right) x(t)+x^{T}\left(t-\tau_{1}\right)\left(R_{3}-R_{1}\right) x\left(t-\tau_{1}\right) \\
& -(1-\dot{\tau}(t)) x^{T}(t-\tau(t)) R_{2} x(t-\tau(t))-x^{T}\left(t-\tau_{2}\right) R_{3} x\left(t-\tau_{2}\right)+y^{T}(t) R_{4} y(t) \\
& +\left[\begin{array}{c}
y(t) \\
f(y(t))
\end{array}\right]^{T} R_{5}\left[\begin{array}{c}
y(t) \\
f(y(t))
\end{array}\right]+y^{T}\left(t-\sigma_{1}\right)\left(R_{6}-R_{4}\right) y\left(t-\sigma_{1}\right) \\
& -(1-\dot{\sigma}(t))\left[\begin{array}{c}
y(t-\sigma(t)) \\
f(y(t-\sigma(t)))
\end{array}\right]^{T} R_{5}\left[\begin{array}{c}
y(t-\sigma(t)) \\
f(y(t-\sigma(t)))
\end{array}\right]-y^{T}\left(t-\sigma_{2}\right) R_{6} y\left(t-\sigma_{2}\right) \\
& \leq x^{T}(t)\left(R_{1}+R_{2}\right) x(t)+x^{T}\left(t-\tau_{1}\right)\left(R_{3}-R_{1}\right) x\left(t-\tau_{1}\right)-\tau_{d} x^{T}(t-\tau(t)) R_{2} x(t-\tau(t)) \\
& -x^{T}\left(t-\tau_{2}\right) R_{3} x\left(t-\tau_{2}\right)+y^{T}(t) R_{4} y(t)+\left[\begin{array}{c}
y(t) \\
f(y(t))
\end{array}\right]^{T} R_{5}\left[\begin{array}{c}
y(t) \\
f(y(t))
\end{array}\right] \\
& +y^{T}\left(t-\sigma_{1}\right)\left(R_{6}-R_{4}\right) y\left(t-\sigma_{1}\right)-\sigma_{d}\left[\begin{array}{c}
y(t-\sigma(t)) \\
f(y(t-\sigma(t)))
\end{array}\right]^{T} R_{5}\left[\begin{array}{c}
y(t-\sigma(t)) \\
f(y(t-\sigma(t)))
\end{array}\right] \\
& -y^{T}\left(t-\sigma_{2}\right) R_{6} y\left(t-\sigma_{2}\right)=\xi^{T}(t) \Pi_{2} \xi(t), \\
& \dot{V}_{3}(t)=\dot{x}^{T}(t)\left(\tau_{1} W_{1}+\tau_{21} W_{2}\right) \dot{x}(t)+\dot{y}^{T}(t)\left(\sigma_{1} W_{3}+\sigma_{21} W_{4}\right) \dot{y}(t)-\tau_{1} \int_{t-\tau_{1}}^{t} \dot{x}(s) W_{1} \dot{x}(s) \mathrm{d} s \\
& -\int_{t-\tau_{2}}^{t-\tau_{1}} x^{T}(s) W_{2} \dot{x}(s) \mathrm{d} s-\sigma_{1} \int_{t-\sigma_{1}}^{t} y^{T}(s) W_{2} \dot{y}(s) \mathrm{d} s-\int_{t-\sigma_{2}}^{t-\sigma_{1}} y^{T}(s) W_{4} \dot{y}(s) \mathrm{d} s \\
& =\xi^{T}(t) \Pi_{3} \xi(t)-\tau_{1} \int_{t-\tau_{1}}^{t} x^{T}(s) W_{1} \dot{x}(s) \mathrm{d} s-\int_{t-\tau_{2}}^{t-\tau_{1}} x^{T}(s) W_{2} \dot{x}(s) \mathrm{d} s \\
& -\sigma_{1} \int_{t-\sigma_{1}}^{t} y^{T}(s) W_{3} \dot{y}(s) \mathrm{d} s-\int_{t-\sigma_{2}}^{t-\sigma_{1}} y^{T}(s) W_{4} \dot{y}(s) \mathrm{d} s,
\end{aligned}
$$


16

Mathematical Problems in Engineering

$$
\begin{aligned}
\dot{V}_{4}(t)= & \dot{x}^{T}(t)\left(\frac{\tau_{1}^{2}}{2} X_{1}+\frac{\tau_{21}^{2}}{2} X_{2}\right) \dot{x}(t)+\dot{y}^{T}(t)\left(\frac{\sigma_{1}^{2}}{2} X_{3}+\frac{\sigma_{21}^{2}}{2} X_{4}\right) \dot{y}(t) \\
& -\int_{t-\tau_{1}}^{t} \int_{\theta}^{t} x^{\cdot T}(s) X_{1} \dot{x}(s) \mathrm{d} s \mathrm{~d} \theta-\int_{t-\tau_{2}}^{t-\tau_{1}} \int_{\theta}^{t-\tau_{1}} x^{\cdot T}(s) X_{2} \dot{x}(s) \mathrm{d} s \mathrm{~d} \theta-\int_{t-\sigma_{1}}^{t} \int_{\theta}^{t} y^{T}(s) X_{3} \dot{y}(s) \mathrm{d} s \mathrm{~d} \theta \\
& -\int_{t-\sigma_{2}}^{t-\sigma_{1}} \int_{\theta}^{t-\sigma_{1}} y^{\cdot T}(s) X_{4} \dot{y}(s) \mathrm{d} s \mathrm{~d} \theta=\xi^{T}(t) \Pi_{4} \xi(t)-\int_{t-\tau_{1}}^{t} \int_{\theta}^{t} x^{\cdot T}(s) X_{1} \dot{x}(s) \mathrm{d} s \mathrm{~d} \theta-\int_{t-\sigma_{1}}^{t} \int_{\theta}^{t} y^{\cdot T}(s) X_{3} \dot{y}(s) \mathrm{d} s \mathrm{~d} \theta \\
& -\int_{t-\tau(t)}^{t-\tau_{1}} \int_{\theta}^{t-\tau_{1}} x^{\cdot T}(s) X_{2} \dot{x}(s) \mathrm{d} s \mathrm{~d} \theta-\int_{t-\tau_{2}}^{t-\tau(t)} \int_{\theta}^{t-\tau(t)} x^{\cdot T}(s) X_{2} \dot{x}(s) \mathrm{d} s \mathrm{~d} \theta-\int_{t-\sigma(t)}^{t-\sigma_{1}} \int_{\theta}^{t-\sigma_{1}} y^{\cdot T}(s) X_{4} \dot{y}(s) \mathrm{d} s \mathrm{~d} \theta \\
& -\int_{t-\sigma_{2}}^{t-\sigma(t)} \int_{\theta}^{t-\sigma(t)} \dot{y}^{T}(s) X_{4} \dot{y}(s) \mathrm{d} s \mathrm{~d} \theta-\tau_{2}(t) \int_{t-\tau(t)}^{t-\tau_{1}} x^{\cdot T}(s) X_{2} \dot{x}(s) \mathrm{d} s-\sigma_{2}(t) \int_{t-\sigma(t)}^{t-\sigma_{1}} y^{T}(s) X_{4} \dot{y}(s) \mathrm{d} s .
\end{aligned}
$$

And

$$
\dot{V}_{J}=\dot{V}_{J 1}(t)+\dot{V}_{J 2}(t)
$$

where

$$
\begin{aligned}
& \dot{V}_{J 1}(t)=\dot{\tau}(t)\left(\int_{t-\tau(t)}^{t-\tau_{1}} \dot{x}^{T}(s) M_{1} \dot{x}(s) \mathrm{d} s \int_{t-\tau_{2}}^{t-\tau(t)} \dot{x}^{T}(s) M_{2} \dot{x}(s) \mathrm{d} s\right) \\
& +\tau_{1}(t)\left(\dot{x}^{T}\left(t-\tau_{1}\right) M_{1} \dot{x}\left(t-\tau_{1}\right)-\tau_{d} \dot{x}^{T}(t-\tau(t)) M_{1} \dot{x}(t-\tau(t))\right) \\
& +\tau_{2}(t)\left(\tau_{d} \dot{x}^{T}(t-\tau(t)) M_{2} \dot{x}(t-\tau(t))-\dot{x}^{T}\left(t-\tau_{2}\right) M_{2} \dot{x}\left(t-\tau_{2}\right)\right) \\
& -2\left(x\left(t-\tau_{1}\right)-x(t-\tau(t))\right)^{T} M_{1}\left(\dot{x}\left(t-\tau_{1}\right)-\tau_{d} \dot{x}(t-\tau(t))\right) \\
& -2\left(x(t-\tau(t))-x\left(t-\tau_{2}\right)\right)^{T} M_{2}\left(\tau_{d} \dot{x}(t-\tau(t))-\dot{x}\left(t-\tau_{2}\right)\right) \\
& +\dot{\tau}(t)\left(\int_{t-\tau(t)}^{t-\tau_{1}} \int_{s}^{t-\tau_{1}} \dot{x}^{T}(u) M_{3} \dot{x}(u) \mathrm{d} u \mathrm{~d} s-\int_{t-\tau_{2}}^{t-\tau(t)} \int_{s}^{t-\tau(t)} \dot{x}^{T}(u) M_{4} \dot{x}(u) \mathrm{d} u \mathrm{~d} s\right) \\
& +\tau_{1}(t)\left(\tau_{1}(t) \dot{x}^{T}\left(t-\tau_{1}\right) M_{3} \dot{x}\left(t-\tau_{1}\right)-\int_{t-\tau(t)}^{t-\tau_{1}} \dot{x}^{T}(s) M_{3} \dot{x}(s) \mathrm{d} s\right) \\
& +\tau_{2}(t)\left(\tau_{d} \tau_{2}(t) \dot{x}^{T}(t-\tau(t)) M_{4} \dot{x}(t-\tau(t))-\int_{t-\tau_{2}}^{t-\tau(t)} \dot{x}^{T}(s) M_{4} \dot{x}(s) \mathrm{d} s\right) \\
& -\frac{1}{\tau_{21}^{2}}\left(\dot{\tau}(t) \eta_{1}^{T}(t) M_{3} \eta_{1}(t)-\dot{\tau}(t) \eta_{2}^{T}(t) M_{4} \eta_{2}(t)+\operatorname{Sym}\left\{\eta_{1}^{T}(t) M_{3} \eta_{5}(t)\right\}+\operatorname{Sym}\left\{\eta_{2}^{T}(t) M_{4} \eta_{6}(t)\right\}\right),
\end{aligned}
$$




$$
\begin{aligned}
\dot{V}_{J 2}(t)= & \dot{\sigma}(t)\left(\int_{t-\sigma(t)}^{t-\sigma_{1}} \dot{y}^{T}(s) N_{1} \dot{y}(s) \mathrm{d} s-\int_{t-\sigma_{2}}^{t-\sigma(t)} \dot{y}^{T}(s) N_{2} \dot{y}(s) \mathrm{d} s\right) \\
& +\sigma_{1}(t)\left(\dot{y}^{T}\left(t-\sigma_{1}\right) N_{1} \dot{y}\left(t-\sigma_{1}\right)-\sigma_{d} \dot{y}^{T}(t-\sigma(t)) N_{1} \dot{y}(t-\sigma(t))\right) \\
& +\sigma_{2}(t)\left(\sigma_{d} \dot{y}^{T}(t-\sigma(t)) N_{2} \dot{y}(t-\sigma(t))-\dot{y}^{T}\left(t-\sigma_{2}\right) N_{2} \dot{y}\left(t-\sigma_{2}\right)\right) \\
& -2\left(y\left(t-\sigma_{1}\right)-y(t-\sigma(t))\right)^{T} N_{1}\left(\dot{y}\left(t-\sigma_{1}\right)-\sigma_{d} \dot{y}(t-\sigma(t))\right) \\
& -2\left(y(t-\sigma(t))-y\left(t-\sigma_{2}\right)\right)^{T} N_{2}\left(\sigma_{d} \dot{y}(t-\sigma(t))-\dot{y}\left(t-\sigma_{2}\right)\right) \\
& +\sigma(t)\left(\int_{t-\sigma(t)}^{t-\sigma_{1}} \int_{s}^{t-\sigma_{1}} \dot{y}^{T}(u) N_{3} \dot{y}(u) \mathrm{d} u \mathrm{~d} s-\int_{t-\sigma_{2}}^{t-\sigma(t)} \int_{s}^{t-\sigma(t)} \dot{y}^{T}(u) N_{4} \dot{y}(u) \mathrm{d} u \mathrm{~d} s\right) \\
& +\sigma_{1}(t)\left(\sigma_{1}(t) \dot{y}^{T}\left(t-\sigma_{1}\right) N_{3} \dot{y}\left(t-\sigma_{1}\right)-\int_{t-\sigma(t)}^{t-\sigma_{1}} \dot{y}^{T}(s) N_{3} \dot{y}(s) \mathrm{d} s\right) \\
& +\sigma_{2}(t)\left(\sigma_{d} \sigma_{2}(t) \dot{y}^{T}(t-\sigma(t)) N_{4} \dot{y}(t-\sigma(t))-\int_{t-\sigma_{2}}^{t-\sigma(t)} \dot{y}^{T}(s) N_{4} \dot{y}(s) \mathrm{d} s\right) \\
& -\frac{1}{\sigma_{21}^{2}}\left(\dot{\sigma}(t) \eta_{3}^{T}(t) N_{3} \eta_{3}(t)-\dot{\sigma}(t) \eta_{4}^{T}(t) N_{4} \eta_{4}(t)+\operatorname{Sym}\left\{\eta_{3}^{T}(t) N_{3} \eta_{7}(t)\right\}+\operatorname{Sym}\left\{\eta_{4}^{T}(t) N_{4} \eta_{8}(t)\right\}\right) .
\end{aligned}
$$

So, the time derivative of $V_{J}(t)$ can be rewritten as follows:

$$
\begin{aligned}
\dot{V}_{J}(t)= & \xi^{T}(t)\left(\Omega_{1}(\tau(t), \dot{\tau}(t))\right)+\Omega_{2}(\sigma(t), \dot{\sigma}(t)) \xi(t)+\dot{\tau}(t) \\
& \cdot\left(\int_{t-\tau(t)}^{t-\tau_{1}} x^{T}(s) M_{1} \dot{x}(s) \mathrm{d} s-\int_{t-\tau_{2}}^{t-\tau(t)} x^{T}(s) M_{2} \dot{x}(s) \mathrm{d} s\right) \\
& +\dot{\tau}(t)\left(\int_{t-\tau(t)}^{t-\tau_{1}} \int_{s}^{t-\tau_{1}} \dot{x}^{T}(u) M_{3} \dot{x}(u) \mathrm{d} u \mathrm{~d} s-\int_{t-\tau_{2}}^{t-\tau(t)} \int_{s}^{t-\tau(t)} \dot{x}^{T}(u) M_{4} \dot{x}(u) \mathrm{d} u \mathrm{~d} s\right) \\
& -\tau_{1}(t) \int_{t-\tau(t)}^{t-\tau_{1}} x^{T}(s) M_{3} \dot{x}(s) \mathrm{d} s-\tau_{2}(t) \int_{t-\tau_{2}}^{t-\tau(t)} x^{T}(s) M_{4} \dot{x}(s) \mathrm{d} s \\
& +\dot{\sigma}(t)\left(\int_{t-\sigma(t)}^{t-\sigma_{1}} y^{T}(s) N_{1} \dot{y}(s) \mathrm{d} s-\int_{t-\sigma_{2}}^{t-\sigma(t)} \dot{y}^{T}(s) N_{2} \dot{y}(s) \mathrm{d} s\right) \\
& +\dot{\sigma}(t)\left(\int_{t-\sigma(t)}^{t-\sigma_{1}} \int_{s}^{t-\sigma_{1}} y^{T}(u) N_{3} \dot{y}(u) \mathrm{d} u \mathrm{~d} s-\int_{t-\sigma_{2}}^{t-\sigma(t)} \int_{s}^{t-\sigma(t)} \dot{y}^{T}(u) N_{4} \dot{y}(u) \mathrm{d} u \mathrm{~d} s\right) \\
& -\sigma_{1}(t) \int_{t-\sigma(t)}^{t-\sigma_{1}} y^{T}(s) N_{3} \dot{y}(s) \mathrm{d} s-\sigma_{2}(t) \int_{t-\sigma_{2}}^{t-\sigma(t)} \dot{y}^{T}(s) N_{4} \dot{y}(s) \mathrm{d} s,
\end{aligned}
$$

where 


$$
\begin{aligned}
\Omega_{1}(\tau(t), \dot{\tau}(t))= & \tau_{1}(t)\left(\dot{x}^{T}\left(t-\tau_{1}\right) M_{1} \dot{x}\left(t-\tau_{1}\right)-\tau_{d} \dot{x}^{T}(t-\tau(t)) M_{1} \dot{x}(t-\tau(t))\right) \\
& +\tau_{2}(t)\left(\tau_{d} \dot{x}^{T}(t-\tau(t)) M_{2} \dot{x}(t-\tau(t))-\dot{x}^{T}\left(t-\tau_{2}\right) M_{2} \dot{x}\left(t-\tau_{2}\right)\right) \\
& -2\left(x\left(t-\tau_{1}\right)-x(t-\tau(t))\right)^{T} M_{1}\left(\dot{x}\left(t-\tau_{1}\right)-\tau_{d} \dot{x}(t-\tau(t))\right) \\
& -2\left(x(t-\tau(t))-x\left(t-\tau_{2}\right)\right)^{T} M_{2}\left(\tau_{d} \dot{x}(t-\tau(t))-\dot{x}\left(t-\tau_{2}\right)\right) \\
& +\tau_{1}^{2}(t) \dot{x}^{T}\left(t-\tau_{1}\right) M_{3} \dot{x}\left(t-\tau_{1}\right)+\tau_{d} \tau_{2}^{2}(t) \dot{x}^{T}(t-\tau(t)) M_{4} \dot{x}(t-\tau(t)) \\
& -\dot{\tau}(t) \eta_{1}^{T}(t) M_{3} \eta_{1}(t)+\dot{\tau}(t) \eta_{2}^{T}(t) M_{4} \eta_{2}(t)-\operatorname{Sym}\left\{\eta_{1}^{T}(t) M_{3} \eta_{5}(t)\right\} \\
& -\operatorname{Sym}\left\{\eta_{2}^{T}(t) M_{4} \eta_{6}(t)\right\}=\Phi_{2} \tau^{2}(t)+\Phi_{1} \tau(t)+\Phi_{0}, \\
\Omega_{2}(\sigma(t), \dot{\sigma}(t))= & \sigma_{1}(t)\left(\dot{y}^{T}\left(t-\sigma_{1}\right) N_{1} \dot{y}\left(t-\sigma_{1}\right)-\sigma_{d} \dot{y}^{T}(t-\sigma(t)) N_{1} \dot{y}(t-\sigma(t))\right) \\
& +\sigma_{2}(t)\left(\sigma_{d} \dot{y}^{T}(t-\sigma(t)) N_{2} \dot{y}(t-\sigma(t))-\dot{y}^{T}\left(t-\sigma_{2}\right) N_{2} \dot{y}\left(t-\sigma_{2}\right)\right) \\
& -2\left(y\left(t-\sigma_{1}\right)-y(t-\sigma(t))\right)^{T} N_{1}\left(\dot{y}\left(t-\sigma_{1}\right)-\sigma_{d} \dot{y}(t-\sigma(t))\right) \\
& -2\left(y(t-\sigma(t))-y\left(t-\sigma_{2}\right)\right)^{T} N_{2}\left(\sigma_{d} \dot{y}(t-\sigma(t))-\dot{y}\left(t-\sigma_{2}\right)\right) \\
& +\sigma_{1}^{2}(t) \dot{y}^{T}\left(t-\sigma_{1}\right) N_{3} \dot{y}\left(t-\sigma_{1}\right)+\sigma_{d} \sigma_{2}^{2}(t) \dot{y}^{T}(t-\sigma(t)) N_{4} \dot{y}(t-\sigma(t)) \\
& -\dot{\sigma}(t) \eta_{3}^{T}(t) N_{3} \eta_{3}(t)+\dot{\sigma}(t) \eta_{4}^{T}(t) N_{4} \eta_{4}(t)-\operatorname{Sym}\left\{\eta_{3}^{T}(t) N_{3} \eta_{7}(t)\right\} \\
& -\operatorname{Sym}\left\{\eta_{4}^{T}(t) N_{4} \eta_{8}(t)\right\}=\Psi_{2} \sigma^{2}(t)+\Psi_{1} \sigma(t)+\Psi_{0} .
\end{aligned}
$$

Utilizing Lemmas 2 and 3, the single integral terms and the double integral terms of $\dot{V}_{3}(t), V_{4}(t)$, and $\dot{V}_{J}(t)$, respectively, can be as follows:

$$
\begin{aligned}
& -\tau \int_{t-\tau_{1}}^{t} x^{T}(s) W_{1} \dot{x}(s) \mathrm{d} s-\sigma_{1} \int_{t-\sigma_{1}}^{t} y^{T}(s) W_{3} \dot{y}(s) \mathrm{d} s-\tau_{2}(t) \int_{t-\tau(t)}^{t-\tau_{1}} x^{T}(s) X_{2} \dot{x}(s) \mathrm{d} s \\
& -\sigma_{2}(t) \int_{t-\sigma(t)}^{t-\sigma_{1}} y^{T}(s) X_{4} \dot{y}(s) \mathrm{d} s-\int_{t-\tau_{1}}^{t} \int_{\theta}^{t} \dot{x}^{T}(s) X_{1} \dot{x}(s) \mathrm{d} s \mathrm{~d} \theta-\int_{t-\sigma_{1}}^{t} \int_{\theta}^{t} \dot{y}^{T}(s) X_{3} \dot{y}(s) \mathrm{d} s \mathrm{~d} \theta \\
& -\tau_{1}(t) \int_{t-\tau(t)}^{t-\tau_{1}} x^{T}(s) M_{3} \dot{x}(s) \mathrm{d} s-\tau_{2}(t) \int_{t-\tau_{2}}^{t-\tau(t)} x^{T}(s) M_{4} \dot{x}(s) \mathrm{d} s-\sigma_{1}(t) \int_{t-\sigma(t)}^{t-\sigma_{1}} \dot{y}^{T}(s) N_{3} \dot{y}(s) \mathrm{d} s \\
& -\sigma_{2}(t) \int_{t-\sigma_{2}}^{t-\sigma(t)} y^{T}(s) N_{4} \dot{y}(s) \mathrm{d} s \leq \xi^{T}(t) \psi^{T} \Pi_{5} \psi \xi(t),
\end{aligned}
$$

where

$$
\begin{aligned}
& \Pi_{5}=\operatorname{diag}\left\{\tilde{W}_{1}, \widetilde{W}_{3}, \tilde{X}_{2}+\tilde{M}_{3}, \tilde{X}_{4}+\tilde{N}_{3}, \tilde{X}_{1}, \tilde{X}_{3}, \tilde{M}_{4}, \tilde{N}_{4}\right\}, \\
& \widetilde{W}_{i}=\operatorname{diag}\left\{W_{i}, 3 W_{i}, 5 W_{i}\right\} \text {, } \\
& \tilde{X}_{i+1}=\operatorname{diag}\left\{X_{i+1}, 3 X_{i+1}, 5 X_{i+1}\right\} \text {, } \\
& \widetilde{X}_{i}=\operatorname{diag}\left\{2 X_{i}, 4 X_{i}\right\}, \quad i=1,3 \text {, } \\
& \tilde{M}_{4}=\operatorname{diag}\left\{M_{4}, 3 M_{4}, 5 M_{4}\right\} \text {, } \\
& \tilde{N}_{4}=\operatorname{diag}\left\{N_{4}, 3 N_{4}, 5 N_{4}\right\} \text {, } \\
& \psi=\operatorname{col}\left\{\psi_{1}, \psi_{2}, \psi_{3}, \psi_{4}, \psi_{5}, \psi_{6}, \psi_{7}, \psi_{8}\right\} \text {. }
\end{aligned}
$$

According to (11), it follows that there exist diagonal matrices $H_{1}$ and $H_{2}$; then,

$$
\begin{aligned}
0 \leq & -2(f(y(t))-L y(t))^{T} H_{1} f(y(t)) \\
& -2(f(y(t-\sigma(t)))-L y(t-\sigma(t)))^{T} H_{2} f(y(t-\sigma(t))) \\
= & \xi^{T}(t) \operatorname{Sym}\left\{\Pi_{6}\right\} \xi(t) .
\end{aligned}
$$

Therefore, combining with (66), (74), (75), and (79), we have 


$$
\begin{aligned}
\dot{V}(t)= & \xi^{T}(t)(\Omega(\tau(t), \sigma(t), \dot{\tau}(t), \dot{\sigma}(t))) \xi(t)-\int_{t-\tau(t)}^{t-\tau_{1}} x^{T}(s)\left(W_{2}-\dot{\tau}(t) M_{1}\right) \dot{x}(s) \mathrm{d} s \\
& -\int_{t-\tau_{2}}^{t-\tau(t)} x^{T}(s)\left(W_{2}-\dot{\tau}(t) M_{2}\right) \dot{x}(s) \mathrm{d} s-\int_{t-\sigma(t)}^{t-\sigma_{1}} y^{T}(s)\left(W_{4}-\dot{\sigma}(t) N_{1}\right) \dot{y}(s) \mathrm{d} s \\
& -\int_{t-\sigma_{2}}^{t-\sigma(t)} y^{T}(s)\left(W_{4}-\dot{\sigma}(t) N_{2}\right) \dot{y}(s) \mathrm{d} s-\int_{t-\tau(t)}^{t-\tau_{1}} \int_{\theta}^{t-\tau_{1}} \dot{x}^{T}(s)\left(X_{2}-\dot{\tau}(t) M_{3}\right) \dot{x}(s) \mathrm{d} s \mathrm{~d} \theta \\
& -\int_{t-\tau_{2}}^{t-\tau(t)} \int_{\theta}^{t-\tau(t)} \dot{x}^{T}(s)\left(X_{2}-\dot{\tau}(t) M_{4}\right) \dot{x}(s) \mathrm{d} s \mathrm{~d} \theta-\int_{t-\sigma(t)}^{t-\sigma_{1}} \int_{\theta}^{t-\sigma_{1}} \dot{y}^{T}(u)\left(X_{4}-\dot{\sigma}\left((t) N_{3}\right) \dot{y}(u) \mathrm{d} u \mathrm{~d} \theta\right. \\
& -\int_{t-\sigma_{2}}^{t-\sigma(t)} \int_{\theta}^{t-\sigma(t)} \dot{y}^{T}(u)\left(X_{4}-\dot{\sigma}\left((t) N_{4}\right) \dot{y}(u) \mathrm{d} u \mathrm{~d} \theta\right.
\end{aligned}
$$

where

$$
\begin{aligned}
\Omega(\tau(t), \sigma(t), \dot{\tau}(t), \dot{\sigma}(t))= & \operatorname{Sym}\left\{\Pi_{1}\right\}+\Pi_{2}+\Pi_{3}+\Pi_{4}+\Pi_{5} \\
& +\operatorname{Sym}\left\{\Pi_{6}\right\}+\Omega_{1}\left(\tau_{1}(t), \tau_{2}(t)\right) \\
& +\Omega_{2}\left(\sigma_{1}(t), \sigma_{2}(t)\right)=\Omega_{x}(\dot{\tau}(t)) \\
& +\Omega_{y}(\dot{\sigma}(t)) .
\end{aligned}
$$

Utilizing Lemmas 5 and 6, then the single and double integrals in (80) are estimated as follows:

$$
\begin{aligned}
& -\left(\int_{t-\tau(t)}^{t-\tau_{1}} x^{T}(s)\left(W_{2}-\dot{\tau}(t) M_{1}\right) \dot{x}(s) \mathrm{d} s+\int_{t-\tau_{2}}^{t-\tau(t)} x^{T}(s)\left(W_{2}-\dot{\tau}(t) M_{2}\right) \dot{x}(s) \mathrm{d} s\right) \\
& \leq-\frac{1}{\tau_{12}} \xi^{T}(t)\left[\begin{array}{c}
E_{1} \\
E_{2}
\end{array}\right]^{T}\left(\mathscr{K}_{1}(\tau(t), \dot{\tau}(t))-\mathscr{H}_{1}(\tau(t), \dot{\tau}(t))\right)\left[\begin{array}{c}
E_{1} \\
E_{2}
\end{array}\right] \xi(t), \\
& -\left(\int_{t-\sigma(t)}^{t-\sigma_{1}} y^{T}(s)\left(W_{4}-\dot{\sigma}(t) N_{1}\right) \dot{y}(s) \mathrm{d} s+\int_{t-\sigma_{2}}^{t-\sigma(t)} y^{T}(s)\left(W_{4}-\dot{\sigma}(t) N_{2}\right) \dot{y}(s) \mathrm{d} s\right) \\
& \leq-\frac{1}{\sigma_{12}} \xi^{T}(t)\left[\begin{array}{c}
E_{3} \\
E_{4}
\end{array}\right]^{T}\left(\mathscr{K}_{2}(\sigma(t), \dot{\sigma}(t))-\mathscr{H}_{2}(\sigma(t), \dot{\sigma}(t))\right)\left[\begin{array}{c}
E_{3} \\
E_{4}
\end{array}\right] \xi(t), \\
& -\left(\int_{t-\tau(t)}^{t-\tau_{1}} \int_{\theta}^{t-\tau_{1}} \dot{x}^{T}(s)\left(X_{2}-\dot{\tau}(t) M_{3}\right) \dot{x}(s) \mathrm{d} s \mathrm{~d} \theta+\int_{t-\tau_{2}}^{t-\tau(t)} \int_{\theta}^{t-\tau(t)} \dot{x}^{T}(s)\left(X_{2}-\dot{\tau}(t) M_{4}\right) \dot{x}(s) \mathrm{d} s \mathrm{~d} \theta\right) \\
& \leq-\xi^{T}(t)\left[\begin{array}{c}
F_{1} \\
F_{2}
\end{array}\right]^{T}\left(\mathscr{K}_{3}(\tau(t), \dot{\tau}(t))-\mathscr{H}_{3}(\tau(t) \dot{\tau}(t))\right)\left[\begin{array}{l}
F_{1} \\
F_{2}
\end{array}\right] \xi(t), \\
& -\left(\int_{t-\sigma(t)}^{t-\sigma_{1}} \int_{\theta}^{t-\sigma_{1}} \dot{y}^{T}(s)\left(X_{4}-\dot{\sigma}(t) N_{3}\right) \dot{y}(s) \mathrm{d} s \mathrm{~d} \theta+\int_{t-\sigma_{2}}^{t-\sigma(t)} \int_{\theta}^{t-\sigma(t)} \dot{y}^{T}(s)\left(X_{4}-\dot{\sigma}(t) N_{4}\right) \dot{y}(s) \mathrm{d} s \mathrm{~d} \theta\right) \\
& \leq-\xi^{T}(t)\left[\begin{array}{c}
F_{3} \\
F_{4}
\end{array}\right]^{T}\left(\mathscr{K}_{4}(\sigma(t), \dot{\sigma}(t))-\mathscr{H}_{4}(\sigma(t), \dot{\sigma}(t))\right)\left[\begin{array}{l}
F_{3} \\
F_{4}
\end{array}\right] \xi(t),
\end{aligned}
$$


where

$$
\begin{aligned}
& \mathscr{K}_{1}(\tau(t), \dot{\tau}(t))=\left[\begin{array}{cc}
\left(1+\frac{\tau_{2}(t)}{\tau_{21}}\right) \overline{\mathscr{W}}_{1} & \frac{\tau_{2}(t)}{\tau_{21}} S_{1}+\frac{\tau_{1}(t)}{\tau_{21}} S_{2} \\
* & \left(1+\frac{\tau_{1}(t)}{\tau_{21}}\right) \overline{\mathscr{W}}_{2}
\end{array}\right], \\
& \mathscr{H}_{1}(\tau(t), \dot{\tau}(t))=\left[\begin{array}{cc}
\frac{\tau_{2}(t)}{\tau_{21}} S_{2} \overline{\mathscr{W}}_{2}^{-1} S_{2}^{T} & 0 \\
0 & \frac{\tau_{1}(t)}{\tau_{21}} S_{1}^{T} \overline{\mathscr{W}}_{1}^{-1} S_{1}
\end{array}\right] \text {, } \\
& \mathscr{K}_{2}(\sigma(t), \dot{\sigma}(t))=\left[\begin{array}{cc}
\left(1+\frac{\sigma_{2}(t)}{\tau_{21}}\right) \overline{\mathscr{W}}_{3} & \frac{\sigma_{2}(t)}{\tau_{21}} S_{3}+\frac{\sigma_{1}(t)}{\tau_{21}} S_{4} \\
* & \left(1+\frac{\sigma_{1}(t)}{\tau_{21}}\right) \overline{\mathscr{W}}_{4}
\end{array}\right], \\
& \mathscr{H}_{2}(\sigma(t), \dot{\sigma}(t))=\left[\begin{array}{cc}
\frac{\sigma_{2}(t)}{\tau_{21}} S_{4} \overline{\mathscr{W}}_{4}^{-1} S_{4}^{T} & 0 \\
0 & \frac{\sigma_{1}(t)}{\tau_{21}} S_{3}^{T} \overline{\mathscr{W}}_{3}^{-1} S_{3}
\end{array}\right] \text {, } \\
& \mathscr{K}_{3}(\tau(t), \dot{\tau}(t))=\left[\begin{array}{cc}
\bar{X}_{1} & 0 \\
0 & \overline{\mathscr{X}}_{2}
\end{array}\right] \text {, } \\
& \mathscr{H}_{3}(\tau(t), \dot{\tau}(t))=\left[\begin{array}{cc}
\tau_{2}^{2}(t) T_{2} \overline{\mathscr{X}}_{2}^{-1} T_{2}^{T} & 0 \\
0 & \tau_{1}^{2}(t) T_{1}^{T} \overline{\mathscr{X}}_{1}^{-1} T_{1}
\end{array}\right] \text {, } \\
& \mathscr{K}_{4}(\sigma(t), \dot{\sigma}(t))=\left[\begin{array}{cc}
\bar{X}_{3} & 0 \\
0 & \bar{X}_{4}
\end{array}\right], \\
& \mathscr{H}_{4}(\sigma(t), \dot{\sigma}(t))=\left[\begin{array}{cc}
\alpha_{2}^{2}(t) F_{4} \overline{\mathscr{X}}_{4}^{-1} F_{4}^{T} & 0 \\
0 & \alpha_{1}^{2}(t) F_{3}^{T} \bar{X}_{3}^{-1} F_{3}
\end{array}\right] \text {, } \\
& \overline{\mathscr{W}}_{1}=\operatorname{diag}\left\{W_{2}-\dot{\tau}(t) M_{1}, 3\left(W_{2}-\dot{\tau}(t) M_{1}\right)\right\} \text {, } \\
& \overline{\mathscr{W}}_{2}=\operatorname{diag}\left\{W_{2}+\dot{\tau}(t) M_{2}, 3\left(W_{2}+\dot{\tau}(t) M_{2}\right)\right\}, \\
& \overline{\mathscr{W}}_{3}=\operatorname{diag}\left\{W_{4}-\dot{\sigma}(t) N_{1}, 3\left(W_{4}-\dot{\sigma}(t) N_{1}\right)\right\}, \\
& \overline{\mathscr{W}}_{4}=\operatorname{diag}\left\{W_{4}+\dot{\sigma}(t) N_{2}, 3\left(W_{4}+\dot{\sigma}(t) N_{2}\right)\right\}, \\
& \bar{X}_{1}=\operatorname{diag}\left\{2\left(X_{2}-\dot{\tau}(t) M_{3}\right), 4\left(W_{2}-\dot{\tau}(t) M_{3}\right)\right\} \text {, } \\
& \bar{X}_{2}=\operatorname{diag}\left\{2\left(X_{2}+\dot{\tau}(t) M_{4}\right), 4\left(X_{2}+\dot{\tau}(t) M_{4}\right)\right\} \text {, } \\
& \bar{X}_{3}=\operatorname{diag}\left\{2\left(X_{4}-\dot{\sigma}(t) N_{3}\right), 4\left(X_{4}-\dot{\sigma}(t) N_{3}\right)\right\}, \\
& \bar{X}_{4}=\operatorname{diag}\left\{2\left(X_{4}+\dot{\sigma}(t) N_{4}\right), 4\left(X_{4}+\dot{\sigma}(t) N_{4}\right)\right\} \text {. }
\end{aligned}
$$


Combining (82)-(84), we have $\dot{V}(t)=\xi^{T}(t)\left(\Upsilon_{x}(\tau(t), \dot{\tau}(t))+\Upsilon_{y}(\sigma(t), \dot{\sigma}(t)) \xi(t)\right)$,

$$
\begin{aligned}
& \Upsilon_{x}(\tau(t), \dot{\tau}(t))=a_{2} \tau^{2}(t)+a_{1} \tau(t)+a_{0} \\
& a_{2}=\Phi_{2}+\left[\begin{array}{c}
F_{1} \\
F_{2}
\end{array}\right]^{T}\left[\begin{array}{cc}
T_{2} \bar{X}_{2}^{-1} T_{2}^{T} & 0 \\
0 & T_{1}^{T} \bar{X}_{1}^{-1} T_{1}
\end{array}\right]\left[\begin{array}{c}
F_{1} \\
F_{2}
\end{array}\right]
\end{aligned}
$$

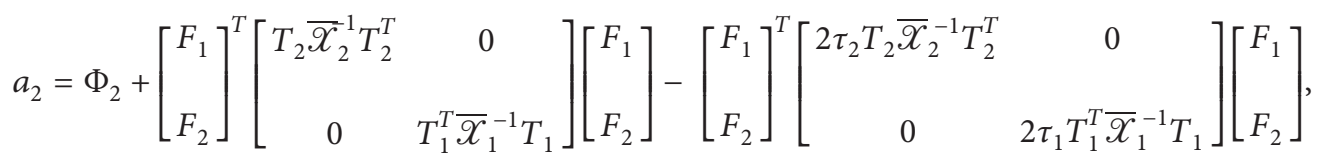

$$
\begin{aligned}
& a_{0}=\Omega_{x}(\dot{\tau}(t))+\Phi_{0}-\left[\begin{array}{l}
E_{1} \\
E_{2}
\end{array}\right]^{T}\left[\begin{array}{cc}
\left(1+\frac{\tau_{2}}{\tau_{21}}\right) \overline{\mathscr{W}}_{1}-\frac{\tau_{2}}{\tau_{21}} S_{2} \overline{\mathscr{W}}_{2}^{-1} S_{2}^{T} & \frac{\tau_{2}}{\tau_{21}} S_{1}+\left(1-\frac{\tau_{2}}{\tau_{21}}\right) S_{2} \\
* & \frac{\tau_{2}}{\tau_{21}} \overline{\mathscr{W}}_{2}-\left(1-\frac{\tau_{2}}{\tau_{21}}\right) S_{1}^{T} \overline{\mathscr{W}}_{1}^{-1} S_{1}
\end{array}\right]\left[\begin{array}{l}
E_{1} \\
E_{2}
\end{array}\right]
\end{aligned}
$$

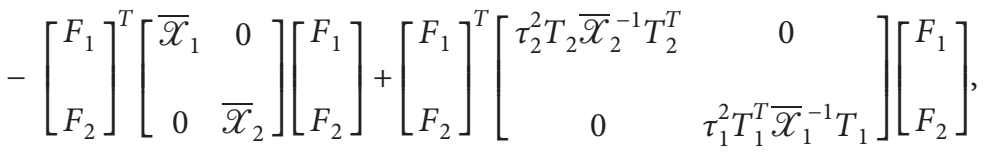

$\Upsilon_{y}(\sigma(t), \dot{\sigma}(t))=b_{2} \sigma^{2}(t)+b_{1} \sigma(t)+b_{0}$

$$
\begin{aligned}
& b_{2}=\Psi_{2}+\left[\begin{array}{c}
F_{3} \\
F_{4}
\end{array}\right]^{T}\left[\begin{array}{cc}
T_{4} \overline{\mathscr{X}}_{4}^{-1} T_{4}^{T} & 0 \\
0 & T_{3}^{T} \overline{\mathscr{X}}_{3}^{-1} T_{3}
\end{array}\right]\left[\begin{array}{c}
F_{3} \\
F_{4}
\end{array}\right] \\
& b_{1}=\Psi_{1}-\frac{1}{\sigma_{21}}\left[\begin{array}{c}
E_{3} \\
E_{4}
\end{array}\right]^{T}\left[\begin{array}{cc}
S_{4} \overline{\mathscr{W}}_{4}^{-1} S_{4}^{T}-\overline{\mathscr{W}}_{3} & S_{4}-S_{3} \\
* & \overline{\mathscr{W}}_{4}-S_{3}^{T} \overline{\mathscr{W}}_{3}^{-1} S_{3}
\end{array}\right]\left[\begin{array}{c}
E_{3} \\
E_{4}
\end{array}\right]-\left[\begin{array}{c}
F_{3} \\
F_{4}
\end{array}\right]^{T}\left[\begin{array}{cc}
2 \sigma_{2} T_{4} \overline{\mathscr{X}}_{4}^{-1} T_{4}^{T} & 0 \\
0 & 2 \sigma_{1} T_{3}^{T} \overline{\mathscr{X}}_{3}^{-1} T_{3}
\end{array}\right]\left[\begin{array}{c}
F_{3} \\
F_{4}
\end{array}\right], \\
& b_{0}=\Omega_{y}(\dot{\sigma}(t))+\Psi_{0}-\left[\begin{array}{c}
E_{3} \\
E_{4}
\end{array}\right]^{T}\left[\begin{array}{cc}
\left(1+\frac{\sigma_{2}}{\sigma_{21}}\right) \overline{\mathscr{W}}_{3}-\frac{\sigma_{2}}{\sigma_{21}} S_{4} \overline{\mathscr{W}}_{4}^{-1} S_{4}^{T} & \frac{\sigma_{2}}{\sigma_{21}} S_{3}+\left(1-\frac{\sigma_{2}}{\sigma_{21}}\right) S_{4} \\
* & \frac{\sigma_{2}}{\sigma_{21}} \overline{\mathscr{W}}_{4}-\left(1-\frac{\sigma_{2}}{\sigma_{21}}\right) S_{3}^{T} \overline{\mathscr{W}}_{3}^{-1} S_{3}
\end{array}\right]\left[\begin{array}{l}
E_{3} \\
E_{4}
\end{array}\right] \\
& -\left[\begin{array}{c}
F_{3} \\
F_{4}
\end{array}\right]^{T}\left[\begin{array}{cc}
\bar{X}_{3} & 0 \\
0 & \bar{X}_{4}
\end{array}\right]\left[\begin{array}{l}
F_{3} \\
F_{4}
\end{array}\right]+\left[\begin{array}{c}
F_{3} \\
F_{4}
\end{array}\right]^{T}\left[\begin{array}{cc}
\sigma_{2}^{2} T_{4} \bar{X}_{4}^{-1} T_{4}^{T} & 0 \\
0 & \sigma_{1}^{2} T_{3}^{T} \bar{X}_{3}^{-1} T_{3}
\end{array}\right]\left[\begin{array}{l}
F_{3} \\
F_{4}
\end{array}\right] .
\end{aligned}
$$


Since $\Upsilon_{x}(\tau(t), \dot{\tau}(t))$ and $\Upsilon_{y}(\sigma(t), \dot{\sigma}(t))$ are convex functions when $2 a_{2}>0$ and $2 b_{2}>0$, it can be guaranteed that if the following conditions hold $\Upsilon_{x}(\tau(t), \dot{\tau}(t))<0$, for all $\tau(t) \in\left[\tau_{1}, \tau_{2}\right]$ when

$$
\begin{array}{r}
\Upsilon_{x}\left(\tau_{1}, \dot{\tau}(t)\right)<0, \\
\Upsilon_{x}\left(\tau_{1}, \dot{\tau}(t)\right)<0, \\
2 a_{2}>0 .
\end{array}
$$

and $\Upsilon_{y}(\sigma(t), \dot{\sigma}(t))<0$, for all $\sigma(t) \in\left[\sigma_{1}, \sigma_{2}\right]$, when

$$
\begin{array}{r}
\Upsilon_{y}\left(\sigma_{1}, \dot{\sigma}(t)\right)<0 \\
\Upsilon_{y}\left(\sigma_{2}, \dot{\sigma}(t)\right)<0, \\
2 b_{2}>0
\end{array}
$$

Thus, it can be concluded that

$$
\Upsilon_{x}(\tau(t), \dot{\tau}(t))+\Upsilon_{y}(\sigma(t), \dot{\sigma}(t))<0
$$

for all $\tau(t) \in\left[\tau_{1}, \tau_{2}\right]$ and $\sigma(t) \in\left[\sigma_{1}, \sigma_{2}\right]$.

Finally, by applying Schur complement to (87), then LMIs are equivalent to (91), which implies $\dot{V}(t)<0$ for a sufficient small scalar $\epsilon>0$. Therefore, if LMIs (45)-(51) hold, then GRNs (10) are asymptotically stable. This completes our proof.

Remark 7. In the proof of Theorem 1 , the function $V_{0}(t)$ is usually shown in $[20,31,35]$ for the proof of sufficient condition which leads to a less conservative stability condition. Although, Li et al. [35] added function $V_{0}(t)$ that related information interval of time-varying delays $\tau(t)$ and $\sigma(t)$, adding information of time-varying delays may reduce conservativeness but the number of decision variables in LMI are more than the original function $V_{0}(t)$. Thus, our Lyapunov functional $V_{0}(t)$ is not necessary to add information related in time-varying delays $\tau(t)$ and $\sigma(t)$.

Remark 8. Utilizing Lemma 5 in double integral terms (84) and (85) affects LMI condition (91) which is in the form of nonlinear time-delayed terms $\tau^{2}(t)$ and $\sigma^{2}(t)$. The stability criterion is obtained unfeasible by MATLAB LMI tools. However, it can be easy to find negative conditions for a quadratic function with time-varying delays by using the convex function [44].

Remark 9. The estimation of the derivative of the new Lyapunov functions $V_{3}(t)$ and $V_{4}(t)$ are considered together which appear in (82)-(85). By employing extended relaxed one integral inequality (Lemma 5) and the extended relaxed double integral inequality (Lemma 6), it ensures that the conservativeness in stability condition is reduced compared with [32, 33]. Moreover, the estimation of the double integral terms in the derivative forms (84) and (85) guarantees our approach to be less conservative than one in [35]. The effectiveness of this method will be demonstrated in numerical simulation.
TABLE 1: The maximum allowable delay bound (MADB) of $\tau(t)$ for different $\tau_{1}$.

\begin{tabular}{lccc}
\hline Methods & 0.1 & 0.5 & 1 \\
\hline Corollary 3.1, [34] & 5.3 & 5.45 & 5.94 \\
Theorem 1, [20] & 5.5 & 5.91 & 6.41 \\
Theorem 1, [35] & 9.26 & 9.66 & 10.16 \\
Theorem 1 & 9.15 & 9.75 & 10.35 \\
\hline
\end{tabular}

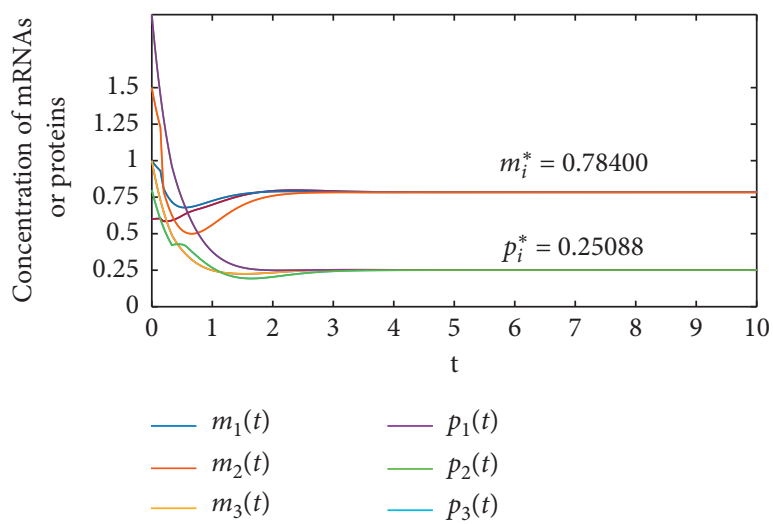

FIgURE 1: The trajectories of mRNAs and proteins.

Remark 10. The stability criterion of Theorem 1 in the form LMIs (45)-(51) can be easy to examine by using LMI toolbox in MATLAB [45].

Remark 11. The improved stability conditions by constructing new Lyapunov functionals are based on LMIs and the dimension of the LMIs depends on the number of the genes in GRNs. Thus, the computational burden problem goes up. This problem is the issue in studying needs of LMI optimization in applied mathematics and the optimization research. Hence, further new techniques are developed to reduce the conservativeness caused by the time delays such as the delay-fractioning approach.

\section{Numerical Example}

In this section, we provide numerical example with a simulation to demonstrate the effectiveness of our results.

Example 1. Consider the genetic regulatory networks (10) with the following parameters [46]:

$$
\begin{aligned}
A & =\operatorname{diag}\{3,3,3\}, \\
C & =\operatorname{diag}\{2.5,2.5,2.5\}, \\
W & =\left[\begin{array}{ccc}
0 & 0 & -2.5 \\
-2.5 & 0 & 0 \\
0 & -2.5 & 0
\end{array}\right] .
\end{aligned}
$$

$D=\operatorname{diag}\{0.8,0.8,0.8\}, \quad f_{i}\left(y_{i}\right)=y_{i}^{2} / 1+y_{i}^{2}, \quad i=1,2,3$, and $K=\operatorname{diag}\{0.65,0.65,0.65\}$.

Assume $\sigma_{1}=0.1, \sigma_{2}=0.3, \tau_{d}=1.5$, and $\sigma_{d}=0.7$, and the maximum allowable delay bound (MADB) of $\tau_{2}$ with 
respect to various $\tau_{1}$ which is obtained in Table 1 . Moreover, the time-varying delays $\tau(t)$ and $\sigma(t)$ are assumed to $\tau(t)=$ $5.4 \sin ^{2}(5 / 18 t)+0.1$ and $\sigma(t)=0.2 \sin ^{2}(3.5 t)+0.1$. So, the trajectories of mRNA and proteins are given in Figure 1 with the initial conditions $m=[0.6,1,1.5]^{T}, t \in[-5.5,0]$ and $p=[1,2,0.8]^{T}, t \in[-0.3,0]$. And the unique equilibrium point $\left(m^{*}, p^{*}\right)$ of $(5)$ is $m^{*}=\left[\begin{array}{lll}0.78 & 0.78 & 0.78\end{array}\right]^{T}$ and $p^{*}=\left[\begin{array}{lll}0.25 & 0.25 & 0.25\end{array}\right]^{T}$.

\section{Conclusions}

In this paper, the stability analysis problem for genetic regulatory networks (GRNs) with time-varying delays is studied. The new Lyapunov functionals have been established for deriving the stability criterion for genetic regulatory networks with time-varying delays to reduce the conservativeness of the stability condition. This paper focuses on the construction of new Lyapunov functionals based on Jensen's inequality and relaxed double integral inequality. By employing Lemmas 5 and 6, new delay dependent sufficient conditions are expressed in the terms of linear matrix inequalities (LMIs) to ensure that it is asymptotically stable for GRNs with time-varying delays. Finally, a numerical example was given to illustrate the effectiveness of the theoretical result and to show less conservativeness than some existing results in the literature.

\section{Data Availability}

The data used to support the findings of this study are available from the corresponding author upon request.

\section{Conflicts of Interest}

The authors declare that there are no conflicts of interest regarding the publication of this paper.

\section{Acknowledgments}

The first author was financially supported by the Science Achievement Scholarship of Thailand (SAST). The second author was financially supported by the Thailand Research Fund (TRF) and Office of the Higher Education Commission (OHEC) (Grant no. MRG6280149). The fourth author was financially supported by the University of Phayao.

\section{References}

[1] S. Huang, "Gene expression profiling, genetic networks, and cellular states: An integrating concept for tumorigenesis and drug discovery," Journal of Molecular Medicine, vol. 77, no. 6, pp. 469-480, 1999.

[2] Z. Wang, H. Wu, J. Liang, J. Cao, and X. Liu, "On modeling and state estimation for genetic regulatory networks with polytopic uncertainties," IEEE Transactions on NanoBioscience, vol. 12, no. 1, pp. 13-20, 2013.

[3] A. Graudenzi, R. Serra, M. Villani, C. Damiani, A. Colacci, and S. A. Kauffman, "Dynamical properties of a Boolean model of gene regulatory network with memory," Journal of Computational Biology, vol. 18, no. 10, pp. 1291-1303, 2011.
[4] P. Smolen, D. A. Baxter, and J. H. Byrne, "Mathematical modeling of gene networks," Neuron, vol. 26, no. 3, pp. 567-580, 2000.

[5] H El Samad, M. Khammash, L. Petzold, and D. Gillespie, "Stochastic modelling of gene regulatory networks," International Journal of Robust and Nonlinear Control, vol. 15, pp. 691-771, 2005.

[6] S. Pandiselvi, R. Raja, J. Cao, G. Rajchakit, and B. Ahmad, "Approximation of state variables for discrete-time stochastic genetic regulatory networks with leakage, distributed and probabilistic measurement delays: A robust stability problem," Advances in Difference Equations, vol. 123, no. 1, 2018.

[7] F. Ren, F. Cao, and J. Cao, "Mittag-Leffler stability and generalized Mittag-Leffler stability of fractional-order gene regulatory networks," Neurocomputing, vol. 160, pp. 185-190, 2015.

[8] L. Chen and K. Aihara, "Stability of genetic regulatory networks with time delay," IEEE Transactions on Circuits and Systems I: Fundamental Theory and Applications, vol. 49, no. 5, pp. 602-608, 2002.

[9] K. Balasundaram, R. Raja, A. Pratap, and S. Chandrasekaran, "Impulsive effects on competitive neural networks with mixed delays: Existence and exponential stability analysis," Mathematics and Computers in Simulation, vol. 155, pp. 290-302, 2019.

[10] C.-K. Zhang, Y. He, L. Jiang, and M. Wu, "Stability analysis for delayed neural networks considering both conservativeness and complexity," IEEE Transactions on Neural Networks and Learning Systems, vol. 27, no. 7, pp. 1486-1501, 2016.

[11] C.-K. Zhang, Y. He, L. Jiang, Q. H. Wu, and M. Wu, "Delaydependent stability criteria for generalized neural networks with two delay components," IEEE Transactions on Neural Networks and Learning Systems, vol. 25, no. 7, pp. 1263-1276, 2014.

[12] A. Pratap, R. Raja, C. Sowmiya, O. Bagdasar, J. Cao, and G. Rajchakit, "Global projective lag synchronization of fractional order memristor based BAM neural networks with mixed time varying delays," Asian Journal of Control, vol. 22, no. 1, pp. 570-583, 2020.

[13] A. Pratap, R. Raja, R. P. Agarwal, and O. Bagdasar, "Multiweighted complex structure on fractional order coupled neural networks with linear coupling delay: A robust synchronization problem," Neural Processing Letters, vol. 51, no. 3, pp. 2453-2479, 2020.

[14] A. Cao, R. P. Raja, R. P. Agarwal, and J. Cao, "Stability analysis and robust synchronization of fractional-order competitive neural networks with different time scales and impulsive perturbations," International Journal of Adaptive Control and Signal Processing, vol. 33, no. 11, pp. 1635-1660, 2019.

[15] A. Pratap, R. Raja, J. Cao, F. A. Rihan, and A. R. Seadawy, "Quasi-pinning synchronization and stabilization of fractional order BAM neural networks with delays and discontinuous neuron activations," Chaos, Solitons and Fractals, vol. 131, Article ID 109491, 2020.

[16] F. Ren and J. Cao, "Asymptotic and robust stability of genetic regulatory networks with time-varying delays," Neurocomputating, vol. 71, no. 4-6, pp. 834-842, 2008.

[17] W. Wang and S. Zhong, "Delay-dependent stability criteria for genetic regulatory networks with time-varying delays and nonlinear disturbance," Communications in Nonlinear Science and Numerical Simulation, vol. 17, no. 9, pp. 3597-3611, 2012.

[18] W. Zhang, J.-A. Fang, and Y. Tang, "Robust stability for genetic regulatory networks with linear fractional 
uncertainties," Communications in Nonlinear Science and Numerical Simulation, vol. 17, no. 4, pp. 1753-1765, 2012.

[19] W. Zhang, J.-a. Fang, and Y. Tang, "Stochastic stability of Markovian jumping genetic regulatory networks with mixed time delays," Applied Mathematics and Computation, vol. 217, no. 17, pp. 7210-7225, 2011.

[20] X. Zhang, L. Wu, and S. Cui, “An improved integral inequality to stability analysis of genetic regulatory networks with interval time-varying delays," IEEE Transactions on Computational Biology and Bioinformatics, vol. 12, no. 2, pp. 398-409, 2015.

[21] X. Zhang, L. Wu, and J. Zou, "Globally asymptotic stability analysis for genetic regulatory networks with mixed delays: An M-matrix-based approach," IEEE/ACM Transactions on Computational Biology and Bioinformatics, vol. 13, no. 1, pp. 135-147, 2016.

[22] L. Li and Y. Yang, "On sampled-data control for stabilization of genetic regulatory networks with leakage delays," Neurocomputing, vol. 151, no. 2, pp. 737-744, 2015.

[23] S. Pandiselvi, R. Raja, Q. Zhu, and G. Rajchakit, "A state estimation $\mathrm{H} \infty$ issue for discrete-time stochastic impulsive genetic regulatory networks in the presence of leakage, multiple delays and Markovian jumping parameters," Journal of the Franklin Institute, vol. 355, no. 5, pp. 2735-2761, 2018.

[24] S. Senthilraj, R. Raja, Q. Zhu, R. Samidurai, and H. Zhou, "Delay-dependent asymptotic stability criteria for genetic regulatory networks with impulsive perturbations," Neurocomputing, vol. 214, pp. 981-990, 2016.

[25] F.-X. Wu, "Global and robust stability analysis of genetic regulatory networks with time-varying delays and parameter uncertainties," IEEE Transactions on Biomedical Circuits and Systems, vol. 5, no. 4, pp. 391-398, 2011.

[26] C. Li, L. Chen, and K. Aihara, "Stability of genetic networks with SUM regulatory logic: Lur'e system and LMI approach," IEEE Transactions on Circuits and Systems I: Regular Papers, vol. 53, no. 11, pp. 2451-2458, 2006.

[27] W. He and J. Cao, "Robust stability of genetic regulatory networks with distributed delay," Cognitive Neurodynamics, vol. 2, no. 4, pp. 355-361, 2008.

[28] W. Wang, S. Kiong Nguang, S. Zhong, and F. Liu, "Exponential convergence analysis of uncertain genetic regulatory networks with time-varying delays," ISA Transactions, vol. 53, no. 5, pp. 1544-1553, 2014.

[29] W. Wang, Y. Wang, S. K. Nguang, S. Zhong, and F. Liu, "Delay partition method for the robust stability of uncertain genetic regulatory networks with time-varying delays," Neurocomputing, vol. 173, pp. 899-911, 2016.

[30] P. G. Park, W. I. Lee, and S. Y. Lee, "Improved stability criteria for linear systems with interval-time varying delays: generalized zero equalities approach," Applied Mathematics and Computation, vol. 292, pp. 336-348, 2017.

[31] Z. Li, D. Chen, Y. Liu, and Y. Zhao, "New delay-dependent stability criteria of genetic regulatory networks subject to time-varying delays," Neurocomputing, vol. 207, pp. 763-771, 2016.

[32] T. H. Lee and J. H. Park, "Improved stability conditions of time-varying delay systems based on new Lyapunov functionals," Journal of the Franklin Institute, vol. 355, no. 3, pp. 1176-1191, 2018.

[33] T. H. Lee, H. M. Trinh, and J. H. Park, "Stability analysis of neural networks with time-varying delay by constructing novel Lyapunov functionals," IEEE Transactions on Neural Networks and Learning Systems, vol. 29, no. 9, pp. 4238-4247, 2018.
[34] W. Wang, S. Zhong, and F. Liu, "New delay-dependent stability criteria for uncertain genetic regulatory networks with time-varying delays," Neurocomputing, vol. 93, pp. 1926, 2012.

[35] F.-D. Li, Q. Zhu, H.-T. Xu, and L. Jiang, "Stability analysis of delayed genetic regulatory networks via a relaxed double integral inequality," Mathematical Problems in Engineering, vol. 2017, pp. 1-16, Article ID 4157256, 2017.

[36] P.-L. Liu, "Robust stability analysis of genetic regulatory network with time delays," ISA Transactions, vol. 52, no. 3, pp. 326-334, 2013.

[37] J. H. Koo, D. H. Ji, S. C. Won, and J. H. Park, “An improved robust delay-dependent stability criterion for genetic regulatory networks with interval time delays," Communications in Nonlinear Science and Numerical Simulation, vol. 17, no. 8, pp. 3399-3405, 2012.

[38] W. Wang, S. Zhong, S. Kiong Nguang, and F. Liu, "Novel delay-dependent stability criterion for uncertain genetic regulatory networks with interval time-varying delays," Neurocomputing, vol. 121, pp. 170-178, 2013.

[39] M. Vadyasagar, Nonlinear System Analysis, Prentice-Hall, Englewood Cliffs, NJ, USA, 2nd edition, 1993.

[40] O. M. Kwon, S. M. Lee, J. H. Park, and E. J. Cha, "New approaches on stability criteria for neural networks with interval time-varying delays," Applied Mathematics and Computation, vol. 218, no. 19, pp. 9953-9964, 2012.

[41] A. Seuret, F. Gouaisbaut, and E. J. Cha, "Wirtinger-based integral inequality: Application to time-delay systems," Automatica, vol. 49, no. 9, pp. 2860-2866, 2013.

[42] C.-K. Zhang, Y. He, L. Jiang, M. Wu, and H.-B. Zeng, "Stability analysis of systems with time-varying delay via relaxed integral inequalities," Systems \& Control Letters, vol. 92, pp. 52-61, 2016.

[43] P. Park, J. W. Ko, and C. Jeong, "Reciprocally convex approach to stability of systems with time-varying delays," Automatica, vol. 47, no. 1, pp. 235-238, 2011.

[44] S. Boyd and L. Vandenberghe, Convex Optimization, Cambridge University Press, Cambridge, UK, 2009.

[45] G. Balas, R. Chaing, A. Packard, and M. Safovov, "Robust control toolbox users guide," MathWorks, 2010.

[46] M. B. Elowitz and S. Leibler, "A synthetic oscillatory network of transcriptional regulators," Nature, vol. 403, pp. 335-338, 2000. 